\title{
Projections of self-similar sets with no separation condition
}

\author{
Ábel Farkas
}

\begin{abstract}
We investigate how the Hausdorff dimension and measure of a self-similar set $K \subseteq$ $\mathbb{R}^{d}$ behave under linear images. This depends on the nature of the group $\mathcal{T}$ generated by the orthogonal parts of the defining maps of $K$. We show that if $\mathcal{T}$ is finite then every linear image of $K$ is a graph directed attractor and there exists at least one projection of $K$ such that the dimension drops under the image of the projection. In general, with no restrictions on $\mathcal{T}$ we establish that $\mathcal{H}^{t}(L \circ O(K))=\mathcal{H}^{t}(L(K))$ for every element $O$ of the closure of $\mathcal{T}$, where $L$ is a linear map and $t=\operatorname{dim}_{H} K$. We also prove that for disjoint subsets $A$ and $B$ of $K$ we have that $\mathcal{H}^{t}(L(A) \cap L(B))=0$. Hochman and Shmerkin showed that if $\mathcal{T}$ is dense in $S O(d, \mathbb{R})$ and the strong separation condition is satisfied then $\operatorname{dim}_{H}(g(K))=\min \left\{\operatorname{dim}_{H} K, l\right\}$ where $g$ is a continuously differentiable map of rank $l$. We deduce the same result without any separation condition and we generalize a result of Eroğlu by obtaining that $\mathcal{H}^{t}(g(K))=0$.
\end{abstract}

\section{Introduction}

\subsection{Overview}

Studying the Hausdorff dimension and measure of orthogonal projections and linear images of sets has a long history. The most fundamental result is that for an analytic subset $K$ of $\mathbb{R}^{d}$

$$
\operatorname{dim}_{H} \Pi_{M}(K)=\min \left\{l, \operatorname{dim}_{H}(K)\right\}
$$

for almost all $l$-dimensional subspaces $M$, where $\operatorname{dim}_{H}$ denotes the Hausdorff dimension and $\Pi_{M}: \mathbb{R}^{d} \longrightarrow M$ denotes the orthogonal projection onto $M$. If $\operatorname{dim}_{H}(K)>l$ then

$$
\mathcal{H}^{l}\left(\Pi_{M}(K)\right)>0
$$

for almost all $l$-dimensional subspaces $M$, where $\mathcal{H}^{s}$ denotes the $s$-dimensional Hausdorff measure. These were proved in the case $d=2, l=1$ by Marstrand [22], and generalized to higher dimensions by Mattila [24]. We call a set $K$ an $s$-set if $0<\mathcal{H}^{s}(K)<\infty$. If $l$ is an integer than we call an $l$-set $K$ irregular if $\mathcal{H}^{l}(K \cap M)=0$ for every differentiable $l$-manifold $M$. It was shown by Besicovitch [2] in the planar case and by Federer [12] in the higher dimensional case that for an $l$-set $K$ where $l$ is an integer

$$
\mathcal{H}^{l}\left(\Pi_{M}(K)\right)=0
$$


for almost all $l$-dimensional subspaces $M$ if and only if $K$ is irregular. If $K$ is not irregular then $\mathcal{H}^{l}\left(\Pi_{M}(K)\right)>0$ for almost all $l$-dimensional subspaces $M$.

While the results above provide information about generic projections they do not give any information about an individual projection or linear image of the set. There are examples that show that the 'exceptional set' for which the conclusions do not hold can be 'big' [22]. Analyzing the image of a set under a particular linear map is more difficult even in simple cases, see for example Kenyon [20] and Hochman [16, Theorem 1.6] who consider the 1-dimensional Sierpinski gasket. Hence we restrict the attention to a certain family of sets, namely we assume $K$ to be a self-similar set.

While studying self-similar sets the 'open set condition' is a convenient assumption that makes the proofs significantly simpler. That is why the case when the open set condition is satisfied is quite well-understood but we know much less in the general situation when no separation condition is assumed. The results in this paper include this general situation. Recent results of Hochman were major breakthrough in studying overlapping self-similar sets. A folklore conjecture is that for a self-similar set $K$ on the $\operatorname{line} \operatorname{dim}_{H} K<\min \{1, s\}$ if and only if exact overlapping occurs among the cylinder sets where $s$ denotes the similarity dimension of $K$. Hochman [16, Theorem 1.5] proves this conjecture when only algebraic parameters occur in the defining maps of $K$. In Example 8.6 we provide a self-similar set $\widehat{K} \subseteq \mathbb{R}$ such that after deleting any number of exact overlaps from the defining maps of $\widehat{K}$ we still have exact overlaps and hence the similarity dimension never realises the Hausdorff dimension of the set even if changing the defining maps.

It is easy to see that if $K$ is a self-similar set with all the defining maps homotheties then every linear image of $K$ is itself a self-similar set. It was asked by Mattila [25, Problem 2] in the planar case "what can be said about the measures $\mathcal{H}^{t}\left(\Pi_{M}(K)\right)$ if $t=\operatorname{dim}_{H}(K)<1$ and the defining maps contain rotations?'. Eroğlu [6] showed that if the open set condition is satisfied and the orthogonal part of one of the defining maps is a rotation of infinite order then $\mathcal{H}^{t}\left(\Pi_{M}(K)\right)=0$ for every line $M$. We generalize this result to higher dimensions and for continuously differentiable maps in place of projections without assuming any separation condition. We obtain results on the structure of linear images of $K$ if the transformation group generated by the orthogonal parts of the defining maps is of finite order. We show that linear images of such self-similar sets are graph directed attractors. We establish an invariance result concerning the Hausdorff measure of the linear images of $K$ in the general case with no restrictions on the orthogonal transformation group. As a consequence of this we conclude that for every linear map into another Euclidean space $L: \mathbb{R}^{d} \longrightarrow \mathbb{R}^{d_{2}}$ where $d_{2}$ is an arbitrary natural number and for disjoint subsets $A$ and $B$ of $K$ we have that $\mathcal{H}^{t}(L(A) \cap L(B))=0$ even if no separation condition is satisfied. In particular, projection of disjoint parts of $K$ are almost disjoint.

Peres and Shmerkin [28, Thorem 5] showed that if the orthogonal part of one of the defining maps is a rotation of infinite order then

$$
\operatorname{dim}_{H} \Pi_{M}(K)=\min \left\{1, \operatorname{dim}_{H}(K)\right\}
$$

for every line $M$. Very recently Hochman and Shmerkin [17, Corollary 1.7] generalized this to higher dimensions for continuously differentiable maps in the strong separation condition case. Using their result and a dimension approximation method we deduce the same conclusion without any separation condition. On the other hand, we show that if the orthogonal 
transformation group generated by the orthogonal parts of the defining maps is of finite order then there exists a projection of $K$ such that the dimension drops under the image of the projection.

\subsection{Definitions and Notations}

A self-similar iterated function system (SS-IFS) in $\mathbb{R}^{d}$ is a finite collection of maps $\left\{S_{i}\right\}_{i=1}^{m}$ from $\mathbb{R}^{d}$ to $\mathbb{R}^{d}$ such that all the $S_{i}$ are contracting similarities. The attractor of the SS-IFS is the unique nonempty compact set $K$ such that $K=\bigcup_{i=1}^{m} S_{i}(K)$. The attractor of an SS-IFS is called a self-similar set.

We say that the SS-IFS $\left\{S_{i}\right\}_{i=1}^{m}$ satisfies the strong separation condition (SSC) if $\bigcup_{i=1}^{m} S_{i}(K)$ is a disjoint union. We say that the SS-IFS $\left\{S_{i}\right\}_{i=1}^{m}$ satisfies the open set condition (OSC) if there exists a nonempty open set $U \subseteq \mathbb{R}^{d}$ such that

$$
\bigcup_{i=1}^{m} S_{i}(U) \subseteq U
$$

and the union is disjoint. It is easy to see that SSC implies OSC.

Let $\left\{S_{i}\right\}_{i=1}^{m}$ be an SS-IFS. Then every $S_{i}$ can be uniquely decomposed as

$$
S_{i}(x)=r_{i} T_{i}(x)+v_{i}
$$

for all $x \in \mathbb{R}^{d}$, where $0<r_{i}<1, T_{i}$ is an orthogonal transformation and $v_{i} \in \mathbb{R}^{d}$ is a translation, for all indices $i$. The unique solution $s$ of the equation

$$
\sum_{i=1}^{m} r_{i}^{s}=1
$$

is called the similarity dimension of the SS-IFS. It is well-known that if the SS-IFS satisfies the OSC then $0<\mathcal{H}^{s}(K)<\infty$. Let $\mathcal{T}$ denote the group generated by the orthogonal transformations $\left\{T_{i}\right\}_{i=1}^{m}$. We call $\mathcal{T}$ the transformation group of the SS-IFS.

We denote the set $\{1,2, \ldots, m\}$ by $\mathcal{I}$. Let $\mathbf{i}=\left(i_{1}, \ldots, i_{k}\right) \in \mathcal{I}^{k}$ i.e. a $k$-tuple of indices. Then we write $S_{\mathbf{i}}=S_{i_{1}} \circ \ldots \circ S_{i_{k}}$ and $K_{\mathbf{i}}=S_{\mathbf{i}}(K)$. Since the similarities are decomposed as in (11) we write $r_{\mathbf{i}}=r_{i_{1}} \cdot \ldots \cdot r_{i_{k}}$ and $T_{\mathbf{i}}=T_{i_{1}} \circ \ldots \circ T_{i_{k}}$. For an overview of the theory of self-similar sets see, for example, [7, 8, 18, 23, 30].

We would like to avoid the singular non-interesting case, when $K$ is a single point, which occurs if and only if every $S_{i}$ has the same fixed point. Hence we make the global assumption throughout the whole paper that $K$ contains at least two points. This implies that there are at least two maps in the SS-IFS, i.e. $m>1$. Hence the similarity dimension of the SS-IFS is strictly positive. It is relevant for us that the assumption that $K$ contains at least two points also implies that $\operatorname{dim}_{H} K>0$ even with no separation condition.

Let $G(\mathcal{V}, \mathcal{E})$ be a directed graph, where $\mathcal{V}=\{1,2, \ldots, q\}$ is the set of vertices and $\mathcal{E}$ is the finite set of directed edges such that for each $i \in \mathcal{V}$ there exists $e \in \mathcal{E}$ starting from $i$. Let $\mathcal{E}_{i, j}$ denote the set of edges from vertex $i$ to vertex $j$ and $\mathcal{E}_{i, j}^{k}$ denote the set of sequences of $k$ edges $\left(e_{1}, \ldots, e_{k}\right)$ which form a directed path from vertex $i$ to vertex $j$. A graph directed iterated function system (GD-IFS) in $\mathbb{R}^{d}$ is a finite collection of maps $\left\{S_{e}: e \in \mathcal{E}\right\}$ from $\mathbb{R}^{d}$ 
to $\mathbb{R}^{d}$ such that all the $S_{e}$ are contracting similarities. The attractor of the GD-IFS is the unique $q$-tuple of nonempty compact sets $\left(K_{1}, \ldots, K_{q}\right)$ such that

$$
K_{i}=\bigcup_{j=1}^{q} \bigcup_{e \in \mathcal{E}_{i, j}} S_{e}\left(K_{j}\right) .
$$

The attractor of a GD-IFS is called a graph directed attractor.

Let $\left\{S_{e}: e \in \mathcal{E}\right\}$ be a GD-IFS. Then every $S_{e}$ can be uniquely decomposed as

$$
S_{e}(x)=r_{e} T_{e}(x)+v_{e}
$$

for all $x \in \mathbb{R}^{d}$, where $0<r_{e}<1, T_{e}$ is an orthogonal transformation and $v_{e} \in \mathbb{R}^{d}$ is a translation, for all edges $e$. Let $A^{(s)}$ be the $q \times q$ matrix with $(i, j)$ th entry given by

$$
A_{i, j}^{(s)}=\sum_{e \in \mathcal{E}_{i, j}} r_{e}^{s} .
$$

For a matrix $A$ let $\rho(A)$ denote the spectral radius of $A$, that is the largest absolute value of the eigenvalues of $A$. The unique solution $s$ of the equation

$$
\rho\left(A^{(s)}\right)=1
$$

is called the similarity dimension of the GD-IFS.

The directed graph $G(\mathcal{V}, \mathcal{E})$ is called strongly connected if for every pair of vertices $i$ and $j$ there exist a directed path from $i$ to $j$ and a directed path from $j$ to $i$. We say that the GD-IFS $\left\{S_{e}: e \in \mathcal{E}\right\}$ is strongly connected if $G(\mathcal{V}, \mathcal{E})$ is strongly connected. For an overview of the theory of graph directed attractors see, for example, [8, 26, 31].

\subsection{Statement of results}

It is well-known that if $K$ is an attractor of an SS-IFS such that $|\mathcal{T}|=1$, where $|$.$| denotes$ the cardinality of a set, then $\Pi_{M}(K)$ is also a self-similar set for every $l$-dimensional subspace $M$. It was shown by Fraser [13, Lemma 2.7] that the vertical and horizontal projections of certain 'box-like' planar self-affine sets are graph directed attractors. We show that, in the case of finite $\mathcal{T}$, similar results can be obtained on the structure of the linear images of self-similar sets.

Theorem 1.1. Let $\left\{S_{i}\right\}_{i=1}^{m}$ be an SS-IFS with attractor $K \subseteq \mathbb{R}^{d}$ of similarity dimension $s$ and $L: \mathbb{R}^{d} \longrightarrow \mathbb{R}^{d_{2}}$ be a linear map. Assume that $\mathcal{T}=\left\{O_{1}, \ldots, O_{q}\right\}$ is a finite group where $q=|\mathcal{T}|$. Then there exists a strongly connected GD-IFS in $\mathbb{R}^{d_{2}}$ with attractor $\left(L \circ O_{1}(K), \ldots, L \circ O_{q}(K)\right)$ such that $s$ is the similarity dimension of this GD-IFS with $T_{e}$ the identity map for all directed edges e, and additionally $\mathcal{H}^{s}\left(L \circ O_{1}(K)\right)=\ldots=$ $\mathcal{H}^{s}\left(L \circ O_{q}(K)\right)$.

Our next result states that if the Hausdorff dimension of $K$ equals its similarity dimension and $\mathcal{T}$ is finite then we can always find a projection such that the dimension drops under the projection. We show this by finding a projection where exact overlapping occurs. We note that the assumption, that the Hausdorff and the similarity dimensions are the same, is weaker than the OSC, see [29, Theorem 1.1]. 
Theorem 1.2. Let $\left\{S_{i}\right\}_{i=1}^{m}$ be an SS-IFS with attractor $K \subseteq \mathbb{R}^{d}$ of similarity dimension s. Assume that $\mathcal{T}$ is finite and let $l \in \mathbb{N}, l<d$. Then there exists an l-dimensional subspace $M \subseteq \mathbb{R}^{d}$ such that $\operatorname{dim}_{H}\left(\Pi_{M}(K)\right)<s$.

We denote the set of all orthogonal transformations of $\mathbb{R}^{d}$ by $\mathbb{O}_{d}$ which can be metricized using the Euclidean operator norm

$$
\|T\|=\sup _{x \in \mathbb{R}^{d},\|x\|=1}\|T x\|,
$$

where $\|y\|$ denotes the Euclidean norm of $y \in \mathbb{R}^{d}$. With this metric $\mathbb{O}_{d}$ is a compact topological group. We denote by $\overline{\mathcal{T}}$ the closure of $\mathcal{T}$ in this topology.

The result of Theorem 1.1, that $\mathcal{H}^{s}\left(L \circ O_{i}(K)\right)=\mathcal{H}^{s}\left(L \circ O_{j}(K)\right)$, suggests the following theorem.

Theorem 1.3. Let $\left\{S_{i}\right\}_{i=1}^{m}$ be an SS-IFS with attractor $K \subseteq \mathbb{R}^{d}$, let $t=\operatorname{dim}_{H}(K)$ and $L: \mathbb{R}^{d} \longrightarrow \mathbb{R}^{d_{2}}$ be a linear map. If $\mathcal{H}^{t}(K)>0$ then

$$
\mathcal{H}^{t}(L \circ O(A))=\frac{\mathcal{H}^{t}(A)}{\mathcal{H}^{t}(K)} \mathcal{H}^{t}(L(K))
$$

for all $A \subseteq K$ and $O \in \overline{\mathcal{T}}$.

We note that the assumption in Theorem 1.3, that $\mathcal{H}^{t}(K)>0$, is again a weaker condition than the OSC (see Example 8.6 and Example 8.7) and the only role of this assumption is that we can divide by $\mathcal{H}^{t}(K)$ in the formula. If $\mathcal{H}^{t}(K)=0$ then $\mathcal{H}^{t}(L(K))=0$ for every linear map $L$. In Example 8.7 we construct a self-similar set $K$ with $0<\mathcal{H}^{t}(K)<$ $\infty$ such that there exists no SS-IFS with attractor $K$ that satisfies the OSC.

Theorem 1.3 has an interesting consequence, that the linear images of disjoint parts of $K$ are 'almost disjoint' even if no separation condition is satisfied.

Corollary 1.4. Let $\left\{S_{i}\right\}_{i=1}^{m}$ be an SS-IFS with attractor $K \subseteq \mathbb{R}^{d}$, let $t=\operatorname{dim}_{H}(K)$, let $L: \mathbb{R}^{d} \longrightarrow \mathbb{R}^{d_{2}}$ be a linear map and $A, B \subseteq K$ be such that $\mathcal{H}^{t}(A \cap B)=0$ and $A$ is $\mathcal{H}^{t}$-measurable. Then $\mathcal{H}^{t}(L(A) \cap L(B))=0$.

In [6] Eroğlu showed that if the transformation group of an SS-IFS in $\mathbb{R}^{2}$ contains a dense set of rotations around the origin then $\mathcal{H}^{s}\left(\Pi_{M}(K)\right)=0$ for all lines $M$, where $s$ denotes the similarity dimension of the SS-IFS. Eroğlu's result does not give any information about the projections when the OSC is not satisfied. Using a different approach we generalize this result to higher dimensions for differentiable maps in place of projections and without any separation condition, with $s$ replaced by the Hausdorff dimension of $K$. Both Eroğlu's and our proof based on finding two or several cylinders and fixed direction in which their projection have large overlap. Eroğlu uses similar arguments to those of Simon and Solomyak [19] to show that the projection measure has infinite upper density almost everywhere, whilst we use a Vitali covering argument to show that the Hausdorff measure of the image must collapse.

Let $0<l \leq d$ be integers and let $G_{d, l}$ denote the Grassmann manifold of $l$-dimensional linear subspaces of $\mathbb{R}^{d}$ equipped with the usual topology (see for example [23, Section 3.9]). 
Theorem 1.5. Let $\left\{S_{i}\right\}_{i=1}^{m}$ be an SS-IFS with attractor $K$, let $t=\operatorname{dim}_{H}(K)$, let $U$ be an open neighbourhood of $K$ and assume that there exists $M \in G_{d, l}$ such that the set $\{O(M): O \in \mathcal{T}\}$ is dense in $G_{d, l}$ for some $1 \leq l<d$. Then $\mathcal{H}^{t}(g(K))=0$ for every continuously differentiable map $g: U \longrightarrow \mathbb{R}^{d_{2}}$ such that $\operatorname{rank}\left(g^{\prime}(x)\right) \leq l$ for every $x \in K$.

If $\operatorname{rank}\left(g^{\prime}(x)\right)=d$ for some $x \in K$ then $g$ is a bi-Lipschitz function between a neigbourhood $V$ of $x$ and $g(V)$ and hence $\mathcal{H}^{t}(g(K))=0$ if and only if $\mathcal{H}^{t}(K)=0$.

We note that the assumption, that $\{O(M): O \in \mathcal{T}\}$ is dense for some $M \in G_{d, l}$, is equivalent to that $\{O(M): O \in \mathcal{T}\}$ is dense for each $M \in G_{d, l}$.

It was shown by Peres and Shmerkin [28, Theorem 5] on the plane under the conditions of Theorem 1.5 that $\operatorname{dim}_{H}\left(\Pi_{M}(K)\right)=\min \{t, 1\}$ for every line $M$. This was generalized to higher dimensions by Hochman and Shmerkin [17, Corollary 1.7] for SS-IFS that satisfies the SSC and the SSC was relaxed by Falconer and Jin [10, Corollary 5.2] to the 'strong variational principle'. We use the result of Hochman and Shmerkin and a dimension approximation method (Proposition 1.8) to deduce the same conclusion without any separation condition.

Theorem 1.6. Let $\left\{S_{i}\right\}_{i=1}^{m}$ be an SS-IFS with attractor $K$, let $t=\operatorname{dim}_{H}(K)$, let $U$ be an open neighbourhood of $K$ and assume that there exists $M \in G_{d, l}$ such that the set $\{O(M): O \in \mathcal{T}\}$ is dense in $G_{d, l}$ for some $1 \leq l<d$. Then $\operatorname{dim}_{H}(g(K))=\min \{t, l\}$ for every continuously differentiable map $g: U \longrightarrow \mathbb{R}^{l}$ such that $\operatorname{rank}\left(g^{\prime}(x)\right)=l$ for some $x \in K$.

We can state a corollary of Theorem 1.6 which applies to $g: U \longrightarrow \mathbb{R}^{d_{2}}$ where $d_{2}$ may be greater than $l$.

Corollary 1.7. Let $\left\{S_{i}\right\}_{i=1}^{m}$ be an SS-IFS with attractor $K$, let $t=\operatorname{dim}_{H}(K)$, let $U$ be an open neighbourhood of $K$ and assume that there exists $M \in G_{d, l}$ such that the set $\{O(M): O \in \mathcal{T}\}$ is dense in $G_{d, l}$ for some $1 \leq l<d$. If $g: U \longrightarrow \mathbb{R}^{d_{2}}$ is a continuously differentiable map such that $\operatorname{rank}\left(g^{\prime}(x)\right)=l$ for every $x \in K$ and either of the following conditions is satisfied

(i) $g \in C^{\infty}$,

(ii) $t \leq l$,

then $\operatorname{dim}_{H}(g(K))=\min \{t, l\}$.

In the planar case $|\mathcal{T}|=\infty$ is equivalent to that $\{O(M): O \in \mathcal{T}\}$ is dense in $G_{2,1}$ for every $M \in G_{2,1}$. Furthermore, it can be easily shown that in the planar case $|\mathcal{T}|=\infty$ also implies that $\mathcal{T}$ contains a rotation of infinite order. Example 8.3 shows that in general $|\mathcal{T}|=\infty$ does not imply either the conclusion of Theorem 1.6 or the conclusion of Theorem 1.5 in higher dimensions.

Example 8.5 shows that neither the conclusion of Theorem 1.6 nor the conclusion of Theorem 1.5 necessarily remain true if we replace $g$ with a Lipschitz function that is a composition of an orthogonal projection and a bi-Lipschitz map.

In [14] Furstenberg introduces the definition of a 'dimension conserving map'. If $f$ : $A \longrightarrow \mathbb{R}^{d_{2}}$ is a Lipschitz map where $A \subseteq \mathbb{R}^{d}$ we say that $f$ is dimension conserving if, for some $\delta \geq 0$,

$$
\delta+\operatorname{dim}_{H}\left\{y \in f(A): \operatorname{dim}_{H}\left(f^{-1}(y)\right) \geq \delta\right\} \geq \operatorname{dim}_{H} A
$$


with that convention that $\operatorname{dim}_{H}(\emptyset)=-\infty$ so that $\delta$ cannot be chosen too large. Furstenberg also introduces 'mini- and micro-sets of a set', and a compact set is defined to be 'homogeneous' if all of its micro-sets are also mini-sets. Furstenberg's main theorem [14, Theorem 6.2] states that the restriction of a linear map to a homogeneous compact set is dimension conserving. He suggests that if $K$ is a self-similar set, $\mathcal{T}$ has only one element and the SSC is satisfied then $K$ is homogeneous. One can show that $K$ is homogeneous even if $\mathcal{T}$ is finite and the SSC is satisfied. Thus for such $K$ the restriction of any linear map to $K$ is dimension conserving even though, by Theorem 1.2 , there must be a projection under which the dimension drops. Theorem 1.6 implies that if $\{O(M): O \in \mathcal{T}\}$ is dense in $G_{d, l}$ where $\operatorname{dim}_{H} K \leq l$, then the restriction of $g$ to $K$ is dimension conserving, where $g$ is a continuously differentiable map of rank $l$.

The following proposition is a useful tool for generalizing results about Hausdorff dimension in case of SSC to the case with no separation condition.

Proposition 1.8. Let $\left\{S_{i}\right\}_{i=1}^{m}$ be an $S S$-IFS with attractor $K$. For all $\varepsilon>0$ there exists an SS-IFS $\left\{\widehat{S}_{i}\right\}_{i=1}^{\widehat{m}}$ that satisfies the SSC with attractor $\widehat{K}$ such that $\widehat{K} \subseteq K, \operatorname{dim}_{H} K-\varepsilon<$ $\operatorname{dim}_{H} \widehat{K}$ and the transformation group $\widehat{\mathcal{T}}$ of $\left\{\widehat{S}_{i}\right\}_{i=1}^{\widehat{m}}$ is dense in $\mathcal{T}$.

The planar case of Proposition 1.8 was known before and was used, for example, in [28, 27]. The proof in the planar case is not difficult and in three dimensions is not more complicated. However, the higher dimensional case is more subtle and the proof relies on Kronecker's simultaneous approximation theorem [15, Theorem 442].

All our results are valid without assuming OSC. The following proposition develops a new tool that serves the role of separation conditions in the proofs. In Section 3 we state two other variants of Proposition [1.9 and we hope that such variants of Proposition 1.9 may help to extend other results to settings without any separation condition. The proof of Proposition 1.9 relies on Vitali's covering theorem [9, Theorem 1.10].

Proposition 1.9. Let $\left\{S_{i}\right\}_{i=1}^{m}$ be an SS-IFS with attractor $K$ and let $t=\operatorname{dim}_{H}(K)$. Let $O \in \overline{\mathcal{T}}$ be arbitrary and $\delta>0$. Then there exists $\mathcal{I}_{\infty} \subseteq \bigcup_{k=1}^{\infty} \mathcal{I}^{k}$ such that $\left\|T_{\mathbf{i}}-O\right\|<\delta$ for all $\mathbf{i} \in \mathcal{I}_{\infty}, K_{\mathbf{i}} \cap K_{\mathbf{j}}=\emptyset$ for $\mathbf{i}, \mathbf{j} \in \mathcal{I}_{\infty}, \mathbf{i} \neq \mathbf{j}$, and $\mathcal{H}^{t}\left(K \backslash\left(\bigcup_{\mathbf{i} \in \mathcal{I}_{\infty}} K_{\mathbf{i}}\right)\right)=0$.

Remark 1.10. Under the assumptions of Proposition 1.9 if $K$ is a $t$-set it follows that $\mathcal{H}^{t}(K)=\sum_{\mathbf{i} \in \mathcal{I}_{\infty}} \mathcal{H}^{t}\left(K_{\mathbf{i}}\right)=\sum_{\mathbf{i} \in \mathcal{I}_{\infty}} r_{\mathbf{i}}^{t} \cdot \mathcal{H}^{t}(K)$, hence

$$
\sum_{\mathbf{i} \in \mathcal{I}_{\infty}} r_{\mathbf{i}}^{t}=1 .
$$

This equation plays the role of (2) in the non-OSC case.

Another advantage of Proposition 1.9 is that we can regard the IFS as one for which the orthogonal part $T_{\mathbf{i}}$ of the maps are approximately the same at any level. This observation helps us to deal with the higher dimensional cases when the rotations do not necessarily commute.

The Hausdorff content of a set $H \subseteq \mathbb{R}^{d}$ is $\mathcal{H}_{\infty}^{t}(H)=\inf \left\{\sum_{i=1}^{\infty} \operatorname{diam}\left(U_{i}\right)^{t}: H \subseteq \bigcup_{i=1}^{\infty} U_{i}\right\}$. The next proposition says that the Hausdorff measure and content of linear images of $K$ coincide. It follows that the Hausdorff measure is upper semi-continuous since the Hausdorff 
content is upper semi-continuous. This observation is essential in the proofs of Theorem 1.3 and Theorem 1.5.

Proposition 1.11. Let $\left\{S_{i}\right\}_{i=1}^{m}$ be an SS-IFS with attractor $K \subseteq \mathbb{R}^{d}$, let $t=\operatorname{dim}_{H}(K)$ and $L: \mathbb{R}^{d} \longrightarrow \mathbb{R}^{d_{2}}$ be a linear map. Then

(i) $\mathcal{H}^{t}(L(K))=\mathcal{H}_{\infty}^{t}(L(K))$,

(ii) for every $\varepsilon>0$ there exists $\delta>0$ such that for every linear map $L_{2}: \mathbb{R}^{d} \longrightarrow \mathbb{R}^{d_{2}}$ with $\left\|L-L_{2}\right\|<\delta$ we have that $\mathcal{H}^{t}\left(L_{2}(K)\right) \leq \mathcal{H}^{t}(L(K))+\varepsilon$.

In particular Proposition 1.11 implies that $\mathcal{H}^{t}\left(\Pi_{M}(K)\right)$ is upper semi-continuous in $M \in$ $G_{d, l}$, in contrast with a result of Hochman and Shmerkin [17, Theorem 1.8] on the lower semi-continuity of the lower Hausdorff dimension of Bernoulli convolutions.

It is well-know that if for a set $H$ with $\mathcal{H}^{t}(H)<\infty$ we have that $\mathcal{H}^{t}(H)=\mathcal{H}_{\infty}^{t}(H)$ then for every $\mathcal{H}^{t}$-measurable subset $A \subseteq H$

$$
\mathcal{H}^{t}(H)=\mathcal{H}^{t}(A)+\mathcal{H}^{t}(H \backslash A) \geq \mathcal{H}_{\infty}^{t}(A)+\mathcal{H}_{\infty}^{t}(H \backslash A) \geq \mathcal{H}_{\infty}^{t}(H)=\mathcal{H}^{t}(H)
$$

so $\mathcal{H}^{t}(A)=\mathcal{H}_{\infty}^{t}(A)$.

It follows that $\mathcal{H}^{t}(B)=\mathcal{H}_{\infty}^{t}(B)$ for every $\mathcal{H}^{t}$-measurable subset $B \subseteq L(K)$. Let $A \subseteq$ $L(K)$ be arbitrary and $B \subseteq L(K)$ be a $\mathcal{H}^{t}$-measurable hull of $A$ (for the definition of $\mathcal{H}^{t}$-measurable hull see Section 2.2$)$. We can further assume that $\operatorname{diam}(B)=\operatorname{diam}(A)$. Then $\mathcal{H}^{t}(A)=\mathcal{H}^{t}(B)=\mathcal{H}_{\infty}^{t}(B) \leq \operatorname{diam}(A)^{t}$. In particular for $L=I d_{\mathbb{R}^{d}}$ we obtain that $\mathcal{H}^{t}(A) \leq \operatorname{diam}(A)^{t}$ for every subset $A \subseteq K$. Thus Proposition 2.8 remains valid with $s$ replaced by $t$.

Proposition 1.11 does not generalise to smooth maps. If $K$ is a 1 -set and $L: \mathbb{R}^{d} \longrightarrow \mathbb{R}$ is such that $L(K)=[0,1]$ (see Example 8.1 for $t=1)$ then $g(x):=(\cos (L(x), \sin (L(x))$ : $\mathbb{R}^{d} \longrightarrow \mathbb{R}^{2}$ is such that $\mathcal{H}^{t}(g(K)) \neq \mathcal{H}_{\infty}^{t}(g(K))$.

\section{Preliminaries}

In this section we summarize the background and preliminary results needed to prove our main results.

Let $H \subset \mathbb{R}^{d}$ and write $\operatorname{dim}_{H}(H), \underline{\operatorname{dim}}_{B}(H), \overline{\operatorname{dim}}_{B}(H)$ and $\operatorname{dim}_{P}(H)$ for the Hausdorff dimension, lower box dimension, upper box dimension and packing dimension of $H$ respectively (for the definitions see for example [7]). We denote the diameter of $H$ by $\operatorname{diam}(H)$. We recall that $\mathcal{H}^{s}(H)$ denotes the $s$-dimensional Hausdorff measure of $H$ and $|A|$ denotes the cardinality of a set $A$. If $x \in \mathbb{R}^{d}$ and $r>0$ then we denote the open ball that is centered at $x$ with radius $r$ by $B(x, r)$. We denote the identity map on $\mathbb{R}^{d}$ by $I d_{\mathbb{R}^{d}}$.

\subsection{Orthogonal transformations}

Let $L: \mathbb{R}^{d} \longrightarrow \mathbb{R}^{d_{2}}$ be a linear map. We recall that the Euclidean operator norm of $L$ is defined as

$$
\|L\|=\sup _{x \in \mathbb{R}^{d},\|x\|=1}\|L x\|
$$

where $\|y\|$ denotes the Euclidean norm of a vector $y$. A linear map $T: \mathbb{R}^{d} \longrightarrow \mathbb{R}^{d}$ is called orthogonal if it preserves the norm, i.e. $\|T(y)\|=\|y\|$ for all $y \in \mathbb{R}^{d}$, hence $\|T\|=1$. If $L$ is 
linear and $T$ is orthogonal then it follows that $\|L\|=\|L \circ T\|$. Similarly if $T: \mathbb{R}^{d_{2}} \longrightarrow \mathbb{R}^{d_{2}}$ is an orthogonal transformation and $L$ is linear as above then $\|L\|=\|T \circ L\|$. We recall that $\mathbb{O}_{d}$ denotes the set of all orthogonal transformations. With the Euclidean operator norm metric $\mathbb{O}_{d}$ is a compact topological group.

Lemma 2.1. If $T_{1}, \ldots, T_{m} \in \mathbb{O}_{d}$ then the semigroup generated by $T_{1}, \ldots, T_{m}$ is dense in the group generated by $T_{1}, \ldots, T_{m}$.

Lemma 2.1 is a consequence of the compactness of $\mathbb{O}_{d}$.

Proposition 2.2. If $T \in \mathbb{O}_{d}$ then for all $N \in \mathbb{N}$ there exists $k \in \mathbb{N}, k \geq N$, such that the group generated by $T^{k}$ is dense in the group generated by $T$.

Proposition 2.2 can be deduced from the fact that every compact abelian Lie group is isomorphic to the product of finitely many circle groups and a finite abelian group. However, we provide an elementary proof relying on Kronecker's simultaneous approximation theorem.

Proof. By [3, Theorem 10.12] we can find an orthonormal basis in $\mathbb{R}^{d}$ with respect to which the matrix form of $T$ is block diagonal such that the blocks are either [1] or [-1] or $B\left(\alpha_{i}\right)=$ $\left[\begin{array}{cc}\cos \left(\alpha_{i}\right) & -\sin \left(\alpha_{i}\right) \\ \sin \left(\alpha_{i}\right) & \cos \left(\alpha_{i}\right)\end{array}\right]$ for some $\alpha_{1}, \ldots, \alpha_{n} \in[0,2 \pi)$. Let $\mathcal{J}=\left\{i \in\{1, \ldots, n\}: B\left(\alpha_{i}\right)\right.$ has finite order $\}$. If $i \in \mathcal{J}$ then let $k_{i}$ be the order of $B\left(\alpha_{i}\right)$. Let $k_{0}=2^{N} \cdot \prod_{i \in \mathcal{J}} k_{i}>N$. Then for any $l \in \mathbb{N}$ it follows that $B\left(\alpha_{i}\right)^{l \cdot k_{0}+1}=B\left(\alpha_{i}\right)$ for all $i \in \mathcal{J},[1]^{l \cdot k_{0}+1}=[1]$ and $[-1]^{l \cdot k_{0}+1}=[-1]$.

Let $\mathcal{A}=\left\{\frac{\alpha_{i}}{2 \pi}: i \in\{1, \ldots, n\} \backslash \mathcal{J}\right\}$. If $\frac{\alpha_{i}}{2 \pi} \in \mathcal{A}$ then $\frac{\alpha_{i}}{2 \pi}$ is irrational. Let $1, \frac{\beta_{1}}{2 \pi}, \ldots, \frac{\beta_{m}}{2 \pi} \in$ $\mathcal{A} \bigcup\{1\}$ be a maximal linearly independent system over $\mathbb{Q}$. Then we can write every $\alpha_{i}$ for $i \in\{1, \ldots, n\} \backslash \mathcal{J}$ in the form $\alpha_{i}=\left(\frac{p_{i, 0}}{q_{i, 0}} \cdot 2 \pi+\sum_{j=1}^{m} \frac{p_{i, j}}{q_{i, j}} \cdot \beta_{j}\right)$ such that $p_{i, j} \in \mathbb{Z}, q_{i, j} \in \mathbb{N}$. Let $M=\max \left\{\left|\frac{p_{i, j}}{q_{i, j}}\right|: i \in\{1, \ldots, n\} \backslash \mathcal{J}, j \in\{0, \ldots, m\}\right\}$, let $\widehat{q}=\prod_{i \in\{1, \ldots, n\} \backslash \mathcal{J}} \prod_{j=1}^{m} q_{i, j}$, let $q=\prod_{i \in\{1, \ldots, n\} \backslash \mathcal{J}} q_{i, 0}$ and let $k=k_{0} \cdot q+1$. Let $\delta>0$ be arbitrary. Then $1,(k-1) \cdot k \cdot \frac{\beta_{1}}{2 \pi}, \ldots,(k-$ $1) \cdot k \cdot \frac{\beta_{m}}{2 \pi}$ is a linearly independent system over $\mathbb{Q}$, hence by Kronecker's simultaneous approximation theorem [15, Theorem 442] we can find $p \in \mathbb{N}, d_{1}, \ldots, d_{m} \in \mathbb{Z}$ such that $\left|p \cdot(k-1) \cdot k \cdot \frac{\beta_{j}}{2 \pi}-(1-k) \cdot \frac{\beta_{j}}{2 \pi}-d_{j}\right|<\frac{\delta}{M \cdot m \cdot 2 \pi}$ for $j=1, \ldots, m$ and we can further assume that $p, d_{1}, \ldots, d_{m}$ are multiples of $\widehat{q}$. It follows that $\left|(p \cdot(k-1)+1) \cdot k \cdot \beta_{j}-\beta_{j}-d_{j} \cdot 2 \pi\right|<$ $\frac{\delta}{M \cdot m}$. By the choice of $q$ and $k$ the numbers defined by $D_{i}=(p \cdot(k-1)+1) \cdot k \cdot \frac{p_{i, 0}}{q_{i, 0}}-\frac{p_{i, 0}}{q_{i, 0}}$ are integers for all $i \in\{1, \ldots, n\} \backslash \mathcal{J}$. Thus

$$
\begin{aligned}
& \left|(p \cdot(k-1)+1) \cdot k \cdot \alpha_{i}-\alpha_{i}-D_{i} \cdot 2 \pi-\sum_{j=1}^{m} \frac{p_{i, j}}{q_{i, j}} \cdot d_{j} \cdot 2 \pi\right| \\
& =\left|\sum_{j=1}^{m} \frac{p_{i, j}}{q_{i, j}}\left((p \cdot(k-1)+1) \cdot k \cdot \beta_{j}-\beta_{j}\right)-\frac{p_{i, j}}{q_{i, j}} \cdot d_{j} \cdot 2 \pi\right| \\
& \leq \sum_{j=1}^{m}\left|\frac{p_{i, j}}{q_{i, j}}\right| \cdot\left|(p \cdot(k-1)+1) \cdot k \cdot \beta_{j}-\beta_{j}-d_{j} \cdot 2 \pi\right|<\delta
\end{aligned}
$$


for $i \in\{1, \ldots, n\} \backslash \mathcal{J}$ and by the choice of $\widehat{q}$ we have that $\sum_{j=1}^{m} \frac{p_{i, j}}{q_{i, j}} \cdot d_{j} \in \mathbb{Z}$. So if we set $z=(p \cdot(k-1)+1)$ then $[1]^{k \cdot z}=[1],[-1]^{k \cdot z}=[-1], B\left(\alpha_{i}\right)^{k \cdot z}=B\left(\alpha_{i}\right)$ for all $i \in \mathcal{J}$ and $B\left(\alpha_{i}\right)^{k \cdot z}=B\left(\gamma_{i}\right)$ for all $i \in\{1, \ldots, n\} \backslash \mathcal{J}$ for some $\gamma_{i} \in(0,2 \pi)$ such that $\left|\gamma_{i}-\alpha_{i}\right|<\delta$.

So we can approximate $T$ by the powers of $T^{k}$, hence we can approximate the powers of $T$ by the powers of $T^{k}$. Thus the group generated by $T^{k}$ is dense in the group generated by $T$.

\section{$2.2 \quad$ Linear images}

Every linear map $L: \mathbb{R}^{d} \longrightarrow \mathbb{R}^{d_{2}}$ is a Lipschitz map with Lipschitz constant $\|L\|$.

Giving the following well-known lemma, which we use without reference throughout the paper.

Lemma 2.3. Let $H \subseteq \mathbb{R}^{d}$ and $L: \mathbb{R}^{d} \longrightarrow \mathbb{R}^{d_{2}}$ be a linear map. Then $\mathcal{H}^{s}(L(H)) \leq$ $\|L\|^{s} \cdot \mathcal{H}^{s}(H)$ and $\operatorname{dim}_{H}(L(H)) \leq \operatorname{dim}_{H}(H)$.

A set $A \subseteq \mathbb{R}^{d}$ is called $\mathcal{H}^{s}$-measurable if $\mathcal{H}^{s}(H)=\mathcal{H}^{s}(H \backslash A)+\mathcal{H}^{s}(H \cap A)$ for any set $H \subseteq \mathbb{R}^{d}$. For an arbitrary set $A$ a $\mathcal{H}^{s}$-measurable set $B$ such that $A \subseteq B$ and $\mathcal{H}^{s}(A)=\mathcal{H}^{s}(B)$ is called a $\mathcal{H}^{s}$-measurable hull of $A$.

Lemma 2.4. For every set $A \subseteq \mathbb{R}^{d}$ there exists a Borel set $B$ that is a $\mathcal{H}^{s}$-measurable hull of $A$.

From [23, Theorem 4.4] it can be deduced that there exists a $\mathcal{H}^{s}$-measurable hull of $A$, that is a $G_{\delta}$ set. So there exists a Borel set $B$ that is a $\mathcal{H}^{s}$-measurable hull of $A$.

Lemma 2.5. For a set $A \subseteq \mathbb{R}^{d}$ such that $\mathcal{H}^{s}(A)<\infty$, let $B$ be a $\mathcal{H}^{s}$-measurable hull of $A$ and $L: \mathbb{R}^{d} \longrightarrow \mathbb{R}^{d_{2}}$ be a linear map. Then $\mathcal{H}^{s}(L(A))=\mathcal{H}^{s}(L(B))$.

Proof. Let $C$ be a $\mathcal{H}^{s}$-measurable hull of $L(A)$ such that $C \subseteq L(B)$. Then $B \cap L^{-1}(C)$ is also a $\mathcal{H}^{s}$-measurable hull of $A$. It follows that $\mathcal{H}^{s}\left(B \cap L^{-1}(C)\right)=\mathcal{H}^{s}(A)=\mathcal{H}^{s}(B)$, thus $\mathcal{H}^{s}\left(B \backslash B \cap L^{-1}(C)\right)=0$. Hence $\mathcal{H}^{s}(L(B) \backslash C)=0$ and so $\mathcal{H}^{s}(L(A))=\mathcal{H}^{s}(L(B))$.

Lemma 2.6. Let $0<l<d$ be integers, $v \in \mathbb{R}^{d}, L: \mathbb{R}^{d} \longrightarrow \mathbb{R}^{d_{2}}$ be a linear map with $\operatorname{rank}(L)=l$ and $\mathcal{T} \subseteq \mathbb{O}_{d}$ be such that there exists $M \in G_{d, l}$ such that the set $\{O(M): O \in \mathcal{T}\}$ is dense in $G_{d, l}$. Then there exists $O_{0} \in \overline{\mathcal{T}}$ such that $L \circ O_{0}(v)=0$.

Proof. It is easy to see that if there exists $M \in G_{d, l}$ such that the set $\{O(M): O \in \mathcal{T}\}$ is dense in $G_{d, l}$ then $\{O(M): O \in \mathcal{T}\}$ is dense in $G_{d, l}$ for every $M \in G_{d, l}$. Let $M \in G_{d, l}$ be such that $M$ is contained in the orthogonal complement of $v$. Since $\operatorname{rank}(L)=l$ it follows that $\operatorname{dim}(\operatorname{Ker}(L))=d-l$. Hence $\operatorname{dim}\left(\operatorname{Ker}(L)^{\perp}\right)=l$. There exists $O_{0} \in \overline{\mathcal{T}}$ such that $O_{0}(M)=\operatorname{Ker}(L)^{\perp}$. Since $v$ is orthogonal to $M$ it follows that $O_{0}(v)$ is orthogonal to $O_{0}(M)=\operatorname{Ker}(L)^{\perp}$. Thus $O_{0}(v) \in \operatorname{Ker}(L)$, so $L \circ O_{0}(v)=0$.

\subsection{Vitali's covering theorem}

Let $H \subset \mathbb{R}^{d}$. A collection of sets $\mathcal{A}$ is called a Vitali cover of $H$ if for each $x \in H, \delta>0$ there exists $A \in \mathcal{A}$ with $x \in A$ and $0<\operatorname{diam}(A)<\delta$. 
Proposition 2.7. Let $H \subset \mathbb{R}^{d}$ be a $\mathcal{H}^{s}$-measurable set with $\mathcal{H}^{s}(H)<\infty$ and $B \subset \mathbb{R}^{d}$ be a closed set with $0<\operatorname{diam}(B)<\infty$ and $0<\mathcal{H}^{s}(B)<\infty$. Let $\mathcal{A}$ be a Vitali cover of $H$ such that every element of $\mathcal{A}$ is similar to $B$ and every element of $\mathcal{A}$ is contained in $H$. Then there exists a disjoint sequence of sets (finite or countable) $A_{1}, A_{2}, \ldots \in \mathcal{A}$ such that $\mathcal{H}^{s}\left(H \backslash\left(\bigcup_{i=1}^{\infty} A_{i}\right)\right)=0$.

Proposition 2.7 follows from a version of Vitalis's covering theorem [9, Theorem 1.10] because $\sum_{i=1}^{\infty} \operatorname{diam}\left(A_{i}\right)^{s}=\infty$ is not possible since by the similarity we have that $\sum_{i=1}^{\infty} \operatorname{diam}\left(A_{i}\right)^{s}=$ $\sum_{i=1}^{\infty} \mathcal{H}^{s}\left(A_{i}\right) \cdot \frac{\operatorname{diam}(B)^{s}}{\mathcal{H}^{s}(B)} \leq \mathcal{H}^{s}(H) \cdot \frac{\operatorname{diam}(B)^{s}}{\mathcal{H}^{s}(B)}<\infty$.

\subsection{Self-similar sets}

Proposition 2.8. Let $\left\{S_{i}\right\}_{i=1}^{m}$ be an SS-IFS with attractor $K$ and $s$ be the similarity dimension of $\left\{S_{i}\right\}_{i=1}^{m}$. Then $\mathcal{H}^{s}(K) \leq \operatorname{diam}(K)^{s}<\infty$.

For details see [18, 5.1 Prop(4)].

Proposition 2.9. Let $\left\{S_{i}\right\}_{i=1}^{m}$ be an SS-IFS with attractor $K$. Then $\operatorname{dim}_{H}(K)=\underline{\operatorname{dim}}_{B}(K)=$ $\overline{\operatorname{dim}}_{B}(K)=\operatorname{dim}_{P}(K)$ and $\mathcal{H}^{t}(K)<\infty$ where $t=\operatorname{dim}_{H}(K)$.

Proposition 2.9 can be deduced by an application of implicit methods [8, Thm 3.2].

Proposition 2.10. Let $\left\{S_{i}\right\}_{i=1}^{m}$ be an SS-IFS with attractor $K$ and let $s$ be the similarity dimension of $\left\{S_{i}\right\}_{i=1}^{m}$. Then the following are equivalent:

(i) $\left\{S_{i}\right\}_{i=1}^{m}$ satisfies the $O S C$

(ii) $\operatorname{dim}_{H}(K)=s$ and $0<\mathcal{H}^{s}(K)<\infty$

(iii) $0<\mathcal{H}^{s}(K)$.

For details see [30].

\subsection{Graph directed attractors}

We say that the GD-IFS $\left\{S_{e}: e \in \mathcal{E}\right\}$ satisfies the strong separation condition (SSC) if

$$
K_{i}=\bigcup_{j=1}^{q} \bigcup_{e \in \mathcal{E}_{i, j}} S_{e}\left(K_{j}\right)
$$

is a disjoint union for each $i \in \mathcal{V}$. We say that the GD-IFS $\left\{S_{e}: e \in \mathcal{E}\right\}$ satisfies the open set condition (OSC) if there exists a $q$-tuple of nonempty open sets $\left(U_{1}, \ldots, U_{q}\right)$ such that

$$
\bigcup_{j=1}^{q} \bigcup_{e \in \mathcal{E}_{i, j}} S_{e}\left(U_{j}\right) \subseteq U_{i}
$$

and the union is disjoint for each $i \in \mathcal{V}$. It is easy to see that SSC implies OSC for GD-IFSs.

Proposition 2.11. Let $\left\{S_{e}: e \in \mathcal{E}\right\}$ be a strongly connected $G D$-IFS with attractor $\left(K_{1}, \ldots, K_{q}\right)$ and let $s$ be the similarity dimension of $\left\{S_{e}: e \in \mathcal{E}\right\}$. Then $\mathcal{H}^{s}\left(K_{i}\right)<\infty$ for $i \in \mathcal{V}$. 
For the details of the proof of Proposition 2.11 see [5, p. 172].

Proposition 2.12. Let $\left\{S_{e}: e \in \mathcal{E}\right\}$ be a strongly connected $G D$-IFS with attractor $\left(K_{1}, \ldots, K_{q}\right)$. Then $\operatorname{dim}_{H}\left(K_{j}\right)=\operatorname{dim}_{H}\left(K_{i}\right)=\underline{\operatorname{dim}}_{B}\left(K_{i}\right)=\overline{\operatorname{dim}}_{B}\left(K_{i}\right)=\operatorname{dim}_{P}\left(K_{i}\right)$ for each $i, j \in \mathcal{V}$ and $\mathcal{H}^{t}\left(K_{i}\right)<\infty$ for each $i \in \mathcal{V}$ where $t=\operatorname{dim}_{H}\left(K_{i}\right)$.

Proposition 2.12 can be deduced by the application of the implicit methods [8, Theorem $3.2]$.

Proposition 2.13. Let $\left\{S_{e}: e \in \mathcal{E}\right\}$ be a strongly connected $G D$-IFS with attractor $\left(K_{1}, \ldots, K_{q}\right)$ and let $s$ be the similarity dimension of $\left\{S_{e}: e \in \mathcal{E}\right\}$. Then the following are equivalent:

(i) $\left\{S_{e}: e \in \mathcal{E}\right\}$ satisfies the $O S C$

(ii) $\operatorname{dim}_{H}\left(K_{i}\right)=s$ and $0<\mathcal{H}^{s}\left(K_{i}\right)<\infty$ for each $i \in \mathcal{V}$

(iii) $0<\mathcal{H}^{s}\left(K_{i}\right)$ for some $i \in \mathcal{V}$.

For the details of the proof of Proposition 2.13] see [31].

\subsection{Irreducible matrices}

Recall that for a GD-IFS $\left\{S_{e}: e \in \mathcal{E}\right\}$ we define $A^{(s)}$ as in (15) $A_{i, j}^{(s)}=\sum_{e \in \mathcal{E}_{i, j}} r_{e}^{s}$. Then for the $k$ th power of $A^{(s)}$ it follows that

$$
\left(A^{(s)}\right)_{i, j}^{k}=\sum_{\mathbf{e} \in \mathcal{E}_{i, j}^{k}} r_{\mathbf{e}}^{s}
$$

for all $i, j \in \mathcal{V}$. Thus $G(\mathcal{V}, \mathcal{E})$ is strongly connected if and only if for all $i, j \in \mathcal{V}$ there exists a positive integer $k$ such that $\left(A^{(s)}\right)_{i, j}^{k}>0$.

A $q \times q$ real matrix $A=\left(A_{i, j}\right)$ is called non-negative and we write $A \geq 0$ if $A_{i, j} \geq 0$ for all $1 \leq i, j \leq q$. If $A_{i, j}>0$ holds for all indices $i, j$ then $A$ is called positive and we write $A>0$. For matrices $A$ and $B$ we write $A \geq B$ if $A-B \geq 0$ and similarly we write $A>B$ if $A-B>0$. Similar definitions and notations apply to vectors in $\mathbb{R}^{q}$.

A non-negative matrix $A \geq 0$ is called irreducible if for every $1 \leq i, j \leq q$ indices there exists a positive integer $k$ such that $\left(A^{k}\right)_{i, j}>0$. We note that $k$ can be chosen such that $k \leq q$ (see for example [1, Lem 1.1.2]). There are several equivalent definitions of irreducible matrices but this definition is convenient for us. For a GD-IFS $\left\{S_{e}: e \in \mathcal{E}\right\}$ we have that $A^{(s)} \geq 0$ and $A^{(s)}$ is irreducible if and only if $G(\mathcal{V}, \mathcal{E})$ is strongly connected. Recall that $\rho(A)$ denotes the spectral radius of $A$. The following theorem is the well-known Perron-Frobenius theorem.

Theorem 2.14. Let $A \geq 0$ be a $q \times q$ irreducible matrix. Then

(i) there exist $y \in \mathbb{R}^{q}, y>0$ and $\lambda_{0} \in \mathbb{R}, \lambda_{0}>0$ such that $A y=\lambda_{0} y$,

(ii) the eigenvalue $\lambda_{0}$ is a simple root of the characteristic polynomial of $A$,

(iii) $\rho(A)=\lambda_{0}$,

(iv) the only non-negative, nonzero eigenvectors of $A$ are the positive scalar multiples of $y$.

For details see [1, Thm 1.4.4]. 
Remark 2.15. Let $\left\{S_{e}: e \in \mathcal{E}\right\}$ be a strongly connected GD-IFS with attractor $\left(K_{1}, \ldots, K_{q}\right)$ and let $s$ be the similarity dimension of $\left\{S_{e}: e \in \mathcal{E}\right\}$. Let $y_{i}=\mathcal{H}^{s}\left(K_{i}\right)$ and $y^{\top}=\left(y_{1}, \ldots, y_{q}\right)$. By [31, Proposition 2] we have $\mathcal{H}^{s}\left(K_{e} \cap K_{f}\right)=0$ for $e, f \in \bigcup_{j=1}^{q} \mathcal{E}_{i, j}, e \neq f$. Hence

$$
y_{i}=\mathcal{H}^{s}\left(K_{i}\right)=\sum_{j=1}^{q} \sum_{e \in \mathcal{E}_{i, j}} \mathcal{H}^{s}\left(K_{e}\right)=\sum_{j=1}^{q} \sum_{e \in \mathcal{E}_{i, j}} r_{e}^{s} \cdot \mathcal{H}^{s}\left(K_{j}\right)=\sum_{j=1}^{q} A_{i, j}^{(s)} \cdot y_{j}
$$

SO

$$
y=A^{(s)} y .
$$

If $\left\{S_{e}: e \in \mathcal{E}\right\}$ satisfies the OSC then by Proposition $2.13 y \in \mathbb{R}^{q}, y>0$. In that case $y$ satisfies Theorem 2.14 with $1=\rho\left(A^{(s)}\right)=\lambda_{0}$.

Corollary 2.16. Let $A \geq 0$ be a $q \times q$ irreducible matrix. If there exists a non-negative, non-zero vector $u \in \mathbb{R}^{q}$ such that $A u=u$ then $\rho(A)=1$.

Corollary 2.16 follows from Theorem 2.14

Lemma 2.17. Let $A \geq B \geq 0$ be $q \times q$ irreducible matrices such that $A \neq B$. Then $\rho(A)>\rho(B)$.

Lemma 2.17 follows from [21, 5.7.5].

It follows from Lemma 2.17 that for a GD-IFS $\rho\left(A^{(s)}\right)$ is strictly decreasing in $s$.

Lemma 2.18. Let $\left\{S_{e}: e \in \mathcal{E}\right\}$ be a strongly connected GD-IFS with similarity dimension $s$ and let $e_{0} \in \mathcal{E}$ such that $\left\{S_{e}: e \in \mathcal{E} \backslash\left\{e_{0}\right\}\right\}$ is a strongly connected GD-IFS with similarity dimension $s_{0}$. Then $s_{0}<s$.

Lemma 2.18 follows from Lemma 2.17.

\section{Proof of Proposition 1.9}

In this section our main goal is to prove Proposition 1.9 which provides an important tool to cope with the later results. Proposition 1.9 is essential in the proofs in Section 5 and Section 6 and plays the role of separation conditions when no separation condition is assumed.

Iterating $K=\bigcup_{i=1}^{m} S_{i}(K)$ gives

$$
K=\bigcup_{\mathbf{i} \in \mathcal{I}^{k}} S_{\mathbf{i}}(K)=\bigcup_{\mathbf{i} \in \mathcal{I}^{k}} K_{\mathbf{i}}
$$

for every positive integer $k$.

Lemma 3.1. Let $\left\{S_{i}\right\}_{i=1}^{m}$ be an SS-IFS with attractor $K$ and $t=\operatorname{dim}_{H}(K)$. Then there exists $\mathcal{J} \subseteq \bigcup_{k=1}^{\infty} \mathcal{I}^{k}$ such that $K_{\mathbf{i}} \cap K_{\mathbf{j}}=\emptyset$ for $\mathbf{i}, \mathbf{j} \in \mathcal{J}, \mathbf{i} \neq \mathbf{j}$ and $\mathcal{H}^{t}\left(K \backslash\left(\bigcup_{\mathbf{i} \in \mathcal{J}} K_{\mathbf{i}}\right)\right)=0$.

Proof. $\mathcal{H}^{t}(K)<\infty$ by Proposition 2.9. Let $\mathcal{A}=\left\{K_{\mathbf{i}}: \mathbf{i} \in \bigcup_{k=1}^{\infty} \mathcal{I}^{k}\right\}$. Then $\mathcal{A}$ is a Vitali cover of $K$ and hence Proposition 2.7 provides a $\mathcal{J}$ with the required properties.

Remark 3.2. In Lemma 3.1 for a fixed $\delta>0$ we can further assume that $\operatorname{diam}\left(K_{\mathbf{i}}\right)<\delta$ for every $\mathbf{i} \in \mathcal{J}$ because in the proof we can take $\mathcal{A}=\left\{K_{\mathbf{i}}: \mathbf{i} \in \bigcup_{k=N}^{\infty} \mathcal{I}^{k}\right\}$ for $N$ large enough. 
Lemma 3.3. Let $O \in \overline{\mathcal{T}}$ and $\delta>0$. Then for each $O_{2} \in \overline{\mathcal{T}}$ there exists $\mathbf{j} \in \bigcup_{k=1}^{\infty} \mathcal{I}^{k}$ such that $\left\|O_{2} \circ T_{\mathbf{j}}-O\right\|<\delta$.

Proof. By Lemma 2.1 we can find $\mathbf{j} \in \bigcup_{k=1}^{\infty} \mathcal{I}^{k}$ such that $\left\|O_{2} \circ T_{\mathbf{j}}-O\right\|=\left\|T_{\mathbf{j}}-O_{2}^{-1} \circ O\right\|<$ $\delta$.

Notation 3.4. For $\mathbf{i}=\left(i_{1}, \ldots, i_{k_{1}}\right), \mathbf{j}=\left(j_{1}, \ldots, j_{k_{2}}\right) \in \bigcup_{k=1}^{\infty} \mathcal{I}^{k}$ let $\mathbf{i} * \mathbf{j}=\left(i_{1}, \ldots, i_{k_{1}}, j_{1}, \ldots, j_{k_{2}}\right)$.

In a metric space $(X, d)$ we call a collection $\mathcal{U}$ of subsets of $X$ a $\delta$-cover for some $\delta>0$, if $\mathcal{U}$ is a cover of $X$ and $\operatorname{diam}(U)<\delta$ for every $U \in \mathcal{U}$.

Proof of Proposition 1.9. Since $\overline{\mathcal{T}}$ is compact there exists a finite open $\frac{\delta}{2}$-cover $\left\{U_{i}\right\}_{i=1}^{q}$ of $\overline{\mathcal{T}}$. Let $\mathcal{V}=\{1, \ldots, q\}$ and for every $i \in \mathcal{V}$ fix $O_{i} \in U_{i} \cap \overline{\mathcal{T}}$. By virtue of Lemma 3.3, for each $i \in \mathcal{V}$ we find $\mathbf{j}_{i} \in \bigcup_{k=1}^{\infty} \mathcal{I}^{k}$ such that $\left\|O_{i} \circ T_{\mathbf{j}_{i}}-O\right\|<\frac{\delta}{2}$. So for every $\mathbf{i} \in \bigcup_{k=1}^{\infty} \mathcal{I}^{k}$ there exists $i \in \mathcal{V}$ such that $T_{\mathbf{i}} \in U_{i}$. Then $\left\|T_{\mathbf{i}}-O_{i}\right\|<\frac{\delta}{2}$ and $\left\|O_{i} \circ T_{\mathbf{j}_{i}}-O\right\|<\frac{\delta}{2}$, thus

$$
\left\|T_{\mathbf{i} * \mathbf{j}_{i}}-O\right\|<\delta
$$

By Proposition $2.9 \mathcal{H}^{t}(K)<\infty$. Let $\mathcal{J} \subseteq \bigcup_{k=1}^{\infty} \mathcal{I}^{k}$ be the set provided by Lemma 3.1. We define a sequence of sets $\mathcal{I}_{1}, \mathcal{I}_{2}, \ldots \subseteq \bigcup_{k=1}^{\infty} \mathcal{I}^{k}$ inductively. Let $\mathcal{I}_{1}=\mathcal{J}$. Given $\mathcal{I}_{n}$ is defined we define $\mathcal{I}_{n+1}$ as follows. For each $\mathbf{i} \in \mathcal{I}_{n}$ we define a set $\mathcal{I}_{n+1, \mathbf{i}}$. If $\left\|T_{\mathbf{i}}-O\right\|<\delta$ then let $\mathcal{I}_{n+1, \mathbf{i}}=\{\mathbf{i}\}$. If $\left\|T_{\mathbf{i}}-O\right\| \geq \delta$ then $T_{\mathbf{i}} \in U_{i}$ for some $i \in \mathcal{V}$ and $\left\{K_{\mathbf{i} * \mathbf{j}}: \mathbf{j} \in \bigcup_{k=1}^{\infty} \mathcal{I}^{k}, K_{\mathbf{i} * \mathbf{j}} \cap K_{\mathbf{i} * \mathbf{j}_{i}}=\emptyset\right\}$ is a Vitali cover of $K_{\mathbf{i}} \backslash K_{\mathbf{i} * \mathbf{j}_{i}}$, hence by Proposition 2.7 there exists $\mathcal{J}_{n+1, \mathbf{i}} \subseteq\left\{\mathbf{j}: \mathbf{j} \in \bigcup_{k=1}^{\infty} \mathcal{I}^{k}, K_{\mathbf{i} * \mathbf{j}} \cap K_{\mathbf{i} * \mathbf{j}_{i}}=\emptyset\right\}$ such that $K_{\mathbf{i} * \mathbf{i}_{1}} \cap K_{\mathbf{i} * \mathbf{i}_{2}}=\emptyset$ for $\mathbf{i}_{1}, \mathbf{i}_{2} \in \mathcal{J}_{n+1, \mathbf{i}}, \mathbf{i}_{1} \neq \mathbf{i}_{2}$, and $\mathcal{H}^{t}\left(\left(K_{\mathbf{i}} \backslash K_{\mathbf{i} * \mathbf{j}_{i}}\right) \backslash\left(\bigcup_{\mathbf{j} \in \mathcal{J}_{n+1, \mathbf{i}}} K_{\mathbf{i} * \mathbf{j}}\right)\right)=0$. Then let $\mathcal{I}_{n+1, \mathbf{i}}=$ $\left\{\mathbf{i} * \mathbf{j}_{i}\right\} \bigcup\left\{\mathbf{i} * \mathbf{j}: \mathbf{j} \in \mathcal{J}_{n+1, \mathbf{i}}\right\}$ and let $\mathcal{I}_{n+1}=\bigcup_{\mathbf{i} \in \mathcal{I}_{n}} \mathcal{I}_{n+1, \mathbf{i}}$

Now we define $\mathcal{I}_{\infty}=\bigcap_{n_{1}=1}^{\infty} \bigcup_{n_{2}=n_{1}}^{\infty} \mathcal{I}_{n_{2}}$. Clearly $K_{\mathbf{i}} \cap K_{\mathbf{j}}=\emptyset$ for $\mathbf{i}, \mathbf{j} \in \mathcal{I}_{\infty}, \mathbf{i} \neq \mathbf{j}$. If $\mathbf{i} \in \mathcal{I}_{n}$ and $\left\|T_{\mathbf{i}}-O\right\| \geq \delta$ then $\mathbf{i} \notin \mathcal{I}_{n+l}$ for every positive integer $l$, hence $\mathbf{i} \notin \mathcal{I}_{\infty}$. So $\left\|T_{\mathbf{i}}-O\right\|<\delta$ for all $\mathbf{i} \in \mathcal{I}_{\infty}$. Let $r_{\min }=\min \left\{r_{\mathbf{j}_{i}}: i \in \mathcal{V}\right\}>0$. Clearly

$$
\mathcal{H}^{t}\left(K \backslash\left(\bigcup_{\mathbf{i} \in \mathcal{I}_{n}} K_{\mathbf{i}}\right)\right)=0
$$

for every positive integer $n$. For $\mathbf{i} \in \mathcal{I}_{n}$ such that $\left\|T_{\mathbf{i}}-O\right\| \geq \delta$ and $T_{\mathbf{i}} \in U_{i}$ for some $i \in \mathcal{V}$ (if there are more than one such $i$ then we choose the one that was used above to define the sequence $\left.\mathcal{J}_{n+1, \mathbf{i}}\right)$ we have that $\left\{\mathbf{j}: \mathbf{i} * \mathbf{j} \in \mathcal{I}_{n+1},\left\|T_{\mathbf{i} * \mathbf{j}}-O\right\| \geq \delta\right\} \subseteq \mathcal{J}_{n+1, \mathbf{i}}$ and $\mathcal{H}^{t}\left(K_{\mathbf{i} * \mathbf{j}_{i}}\right)=$ $r_{\mathbf{j}_{i}}^{t} \mathcal{H}^{t}\left(K_{\mathbf{i}}\right) \geq r_{\min }^{t} \mathcal{H}^{t}\left(K_{\mathbf{i}}\right)$, and in the mean time $\left\|T_{\mathbf{i} * \mathbf{j}_{i}}-O\right\|<\delta$ by (9). Therefore $\mathcal{I}_{n+1} \backslash \mathcal{I}_{\infty} \subseteq$ $\bigcup_{\mathbf{i} \in \mathcal{I}_{n} \backslash \mathcal{I}_{\infty}}\left\{\mathbf{i} * \mathbf{j}: \mathbf{j} \in \mathcal{J}_{n+1, \mathbf{i}}\right\}$ and

$$
\begin{aligned}
\mathcal{H}^{t}\left(\bigcup_{\mathbf{i} \in \mathcal{I}_{n+1} \backslash \mathcal{I}_{\infty}} K_{\mathbf{i}}\right) & \leq \sum_{\mathbf{i} \in \mathcal{I}_{n} \backslash \mathcal{I}_{\infty}} \sum_{\mathbf{j} \in \mathcal{J}_{n+1, \mathbf{i}}} \mathcal{H}^{t}\left(K_{\mathbf{i} * \mathbf{j}}\right) \leq \sum_{\mathbf{i} \in \mathcal{I}_{n} \backslash \mathcal{I}_{\infty}}\left(\mathcal{H}^{t}\left(K_{\mathbf{i}}\right)-r_{\min }^{t} \mathcal{H}^{t}\left(K_{\mathbf{i}}\right)\right) \\
& =\sum_{\mathbf{i} \in \mathcal{I}_{n} \backslash \mathcal{I}_{\infty}}\left(1-r_{\min }^{t}\right) \cdot \mathcal{H}^{t}\left(K_{\mathbf{i}}\right)=\left(1-r_{\min }^{t}\right) \cdot \mathcal{H}^{t}\left(\bigcup_{\mathbf{i} \in \mathcal{I}_{n} \backslash \mathcal{I}_{\infty}} K_{\mathbf{i}}\right) .
\end{aligned}
$$


Hence $\mathcal{H}^{t}\left(\bigcup_{\mathbf{i} \in \mathcal{I}_{n+1} \backslash \mathcal{I}_{\infty}} K_{\mathbf{i}}\right) \leq\left(1-r_{\text {min }}^{t}\right)^{n} \cdot \mathcal{H}^{t}\left(\bigcup_{\mathbf{i} \in \mathcal{I}_{1} \backslash \mathcal{I}_{\infty}} K_{\mathbf{i}}\right)$ for all $n \in \mathbb{N}$ and combined with (10) we get that $\mathcal{H}^{t}\left(\bigcup_{\mathbf{i} \in \mathcal{I}_{n+1} \cap \mathcal{I}_{\infty}} K_{\mathbf{i}}\right) \geq\left(1-\left(1-r_{\text {min }}^{t}\right)^{n}\right) \cdot \mathcal{H}^{t}(K)$. Thus $\mathcal{H}^{t}\left(\bigcup_{\mathbf{i} \in \mathcal{I}_{\infty}} K_{\mathbf{i}}\right) \geq$ $\mathcal{H}^{t}(K)$ and so $\mathcal{H}^{t}\left(K \backslash\left(\bigcup_{\mathbf{i} \in \mathcal{I}_{\infty}} K_{\mathbf{i}}\right)\right)=0$.

Corollary 3.5. Let $\left\{S_{i}\right\}_{i=1}^{m}$ be an SS-IFS with attractor $K$ and let $t=\operatorname{dim}_{H}(K)$. Assume that $\mathcal{T}$ is a finite group and let $O \in \mathcal{T}$ be arbitrary. Then there exists $\mathcal{I}_{\infty} \subseteq \bigcup_{k=1}^{\infty} \mathcal{I}^{k}$ such that $T_{\mathbf{i}}=O$ for all $\mathbf{i} \in \mathcal{I}_{\infty}, K_{\mathbf{i}} \cap K_{\mathbf{j}}=\emptyset$ for $\mathbf{i}, \mathbf{j} \in \mathcal{I}_{\infty}, \mathbf{i} \neq \mathbf{j}$ and $\mathcal{H}^{t}\left(K \backslash\left(\bigcup_{\mathbf{i} \in \mathcal{I}_{\infty}} K_{\mathbf{i}}\right)\right)=0$.

Proof. Let $q=|\mathcal{T}|, \mathcal{T}=\left\{O_{1}, \ldots, O_{q}\right\}, \mathcal{V}=\{1, \ldots, q\}$ and $O=O_{i}$ for some $i \in \mathcal{V}$. Then let $\delta=\min _{j \in \mathcal{V}, j \neq i}\left\|O_{j}-O\right\|>0$. By Proposition 1.9 there is an $\mathcal{I}_{\infty}$ such that $\left\|T_{\mathbf{i}}-O\right\|<\delta$ and so $T_{\mathbf{i}}=O$ for all $\mathbf{i} \in \mathcal{I}_{\infty}$.

Proposition 3.6. Let $\left\{S_{i}\right\}_{i=1}^{m}$ be an SS-IFS with attractor $K$ and let $t=\operatorname{dim}_{H}(K)$. Let $O \in \overline{\mathcal{T}}$ be arbitrary and let $\mathbf{i}_{1}, \ldots, \mathbf{i}_{n} \in \bigcup_{k=1}^{\infty} \mathcal{I}^{k}$ be such that $\bigcup_{i=1}^{n} K_{\mathbf{i}_{i}}$ is a disjoint union and let $\delta>0$. Then there exists $\mathcal{I}_{\infty} \subseteq \bigcup_{k=1}^{\infty} \mathcal{I}^{k}$ such that $\left\|T_{\mathbf{i}}-O\right\|<\delta$ for all $\mathbf{i} \in \mathcal{I}_{\infty}$, with $\bigcup_{\mathbf{i} \in \mathcal{I}_{\infty}} \bigcup_{i=1}^{n} K_{\mathbf{i} * \mathbf{i}_{i}}$ a disjoint union and $\mathcal{H}^{t}\left(K \backslash\left(\bigcup_{\mathbf{i} \in \mathcal{I}_{\infty}} \bigcup_{i=1}^{n} K_{\mathbf{i} * \mathbf{i}_{i}}\right)\right)=0$.

The proof of Proposition 3.6 is similar to the proof of Proposition 1.9 with the difference that if we have $\mathbf{i} \in \mathcal{I}_{n}$ at a level such that $\left\|T_{\mathbf{i}}-O\right\|<\delta$ then we keep the pieces $K_{\mathbf{i} * \mathbf{i}_{i}}$ from the next level on and again cover the rest of $K_{\mathbf{i}}$ on the next level.

\section{Iterated function systems with finite transformation groups}

In this section we deal with the case when $\mathcal{T}$ is finite. First, using a natural construction of a GD-IFS we verify Theorem 1.1. Then we prove Theorem 1.2.

Proof of Theorem 1.1. We need to construct a directed graph $G(\mathcal{V}, \mathcal{E})$ and a GD-IFS $\left\{S_{e}: e \in \mathcal{E}\right\}$ that satisfies the theorem. Let $\mathcal{V}$ be the set $\{1,2, \ldots, q\}$. For $i, j \in \mathcal{V}$ and for $n \in \mathcal{I}$ we draw a directed edge $e_{i, j}^{n}$ from $i$ to $j$ if $O_{i} \circ T_{n}=O_{j}$. Then let $\mathcal{E}=\left\{e_{i, j}^{n}: i, j \in \mathcal{V}, n \in \mathcal{I}, O_{i} \circ T_{n}=O_{j}\right\}$.

For $i, j \in \mathcal{V}$ and $n \in \mathcal{I}$ such that $O_{i} \circ T_{n}=O_{j}$, i.e. $e_{i, j}^{n}=e \in \mathcal{E}$, we write $v_{e}=v_{e_{i, j}^{n}}=$ $L \circ O_{i}\left(v_{n}\right), r_{e}=r_{e_{i, j}^{n}}=r_{n}$ and let $S_{e}: \mathbb{R}^{d_{2}} \longrightarrow \mathbb{R}^{d_{2}}$ be the map

$$
S_{e}(x)=S_{e_{i, j}^{n}}(x)=r_{e} \cdot x+v_{e}
$$

Let $\left\{S_{e}: e \in \mathcal{E}\right\}$ be the GD-IFS on the graph $G(\mathcal{V}, \mathcal{E})$. Since $K=\bigcup_{n=1}^{m} S_{n}(K)$, for $i \in \mathcal{V}$,

$$
\begin{aligned}
L\left(O_{i}(K)\right) & =\bigcup_{n=1}^{m} L \circ O_{i} \circ S_{n}(K)=\bigcup_{n=1}^{m} r_{n} \cdot L \circ O_{i} \circ T_{n}(K)+L \circ O_{i}\left(v_{n}\right) \\
& =\bigcup_{n=1}^{m} \bigcup_{j \in \mathcal{V}, O_{i} \circ T_{n}=O_{j}} r_{n} \cdot L \circ O_{j}(K)+L \circ O_{i}\left(v_{n}\right)=\bigcup_{j=1}^{q} \bigcup_{e \in \mathcal{E}_{i, j}} S_{e}\left(L \circ O_{j}(K)\right)
\end{aligned}
$$

and this shows that the $q$-tuple $\left(L \circ O_{1}(K), \ldots, L \circ O_{q}(K)\right)$ is the attractor of $\left\{S_{e}: e \in \mathcal{E}\right\}$. 
Let us show that the the graph $G(\mathcal{V}, \mathcal{E})$ is strongly connected. Let $i, j \in \mathcal{V}$ be arbitrary. Then $O_{i}^{-1} \circ O_{j} \in \mathcal{T}$ and since $\mathcal{T}$ is generated by the transformations $\left\{T_{i}\right\}_{i=1}^{m}$ and each $T_{i}$ has finite order there exists $\mathbf{i}=\left(i_{1}, \ldots, i_{k}\right) \in \mathcal{I}^{k}$ such that $T_{\mathbf{i}}=T_{i_{1}} \circ \ldots \circ T_{i_{k}}=O_{i}^{-1} \circ O_{j}$. Let $\mathbf{j}=\left(j_{1}, \ldots, j_{k}, j_{k+1}\right) \in \mathcal{V}^{k+1}$ such that $j_{1}=i$ and $O_{j_{n}} \circ T_{i_{n}}=O_{j_{n+1}}$ for $1 \leq n \leq k$. This shows that there exists a $k$ step long directed path from $i$ to $j$, that visits vertices $i=j_{1}, \ldots, j_{k}, j_{k+1}=j$ in order. So the graph $G(\mathcal{V}, \mathcal{E})$ is strongly connected.

Let $u=(1, \ldots, 1)^{\top} \in \mathbb{R}^{q}$ be the vector with each coordinate 1 . For the GD-IFS $\left\{S_{e}: e \in \mathcal{E}\right\}$ the matrix $A^{(s)}$ is defined as in (55) $A_{i, j}^{(s)}=\sum_{e \in \mathcal{E}_{i, j}} r_{e}^{s}$, hence the $i$ th coordinate of the vector $A^{(s)} u$ is

$$
\left(A^{(s)} u\right)_{i}=\sum_{j=1}^{q} A_{i, j}^{(s)}=\sum_{j=1}^{q} \sum_{e \in \mathcal{E}_{i, j}} r_{e}^{s}=\sum_{j=1}^{q} \sum_{n=1}^{m} \sum_{e_{i, j}^{n} \in \mathcal{E}_{i, j}} r_{e_{i, j}^{n}}^{s}=\sum_{n=1}^{m} r_{n}^{s}=1
$$

using that $s$ is the similarity dimension of the SS-IFS $\left\{S_{i}\right\}_{i=1}^{m}$. So $u$ is a non-negative, non-zero eigenvector of the irreducible matrix $A^{(s)}$ with eigenvalue 1 . Thus $\rho\left(A^{(s)}\right)=1$ by Corollary 2.16 and hence the similarity dimension of the GD-IFS $\left\{S_{e}: e \in \mathcal{E}\right\}$ is $s$.

Let $y_{i}=\mathcal{H}^{s}\left(L \circ O_{i}(K)\right)$ and $y^{\top}=\left(y_{1}, \ldots, y_{q}\right)$. If the GD-IFS $\left\{S_{e}: e \in \mathcal{E}\right\}$ does not satisfy the OSC then $\mathcal{H}^{s}\left(L \circ O_{1}(K)\right)=\ldots=\mathcal{H}^{s}\left(L \circ O_{q}(K)\right)=0$ by Proposition 2.13, If the GD-IFS $\left\{S_{e}: e \in \mathcal{E}\right\}$ satisfies the OSC then $0<\mathcal{H}^{s}\left(L \circ O_{i}(K)\right)<\infty$ for each $i \in \mathcal{V}$ by Proposition 2.13, hence $y \in \mathbb{R}^{q}, y>0$ and by Remark 2.15 $A^{(s)} y=y$. So $y$ is a positive scalar multiple of $u$ by Theorem 2.14 (iv). So $y=\mathcal{H}^{s}\left(L \circ O_{i}(K)\right) \cdot u$ for each $i \in \mathcal{V}$ and hence $\mathcal{H}^{s}\left(L \circ O_{1}(K)\right)=\ldots=\mathcal{H}^{s}\left(L \circ O_{q}(K)\right)$.

Theorem 1.2 states that we can always find a projection such that the dimension drops under the image of the projection. We show this by finding a projection where exact overlapping occurs.

Proof of Theorem 1.8. We can assume that $l=d-1$ because if $M \in G_{d, d-1}$ such that $\operatorname{dim}_{H}\left(\Pi_{M}(K)\right)<s$ and $N$ is a subspace contained in $M$ then $\Pi_{N}=\Pi_{N} \circ \Pi_{M}$ hence $\operatorname{dim}_{H}\left(\Pi_{N}(K)\right)<s$. Let $\mathcal{T}=\left\{O_{1}, \ldots, O_{q}\right\}$ where $q=|\mathcal{T}|$ and let $\mathcal{V}=\{1,2, \ldots, q\}$. We may assume that $T_{1}=T_{2}=I d_{\mathbb{R}^{d}}$ because if we iterate the IFS $q$ times then we obtain the SS-IFS $\left\{S_{\mathbf{i}}: \mathbf{i} \in \mathcal{I}^{q}\right\}$. The similarity dimension of this new SS-IFS is $s$, the attractor of it is $K$ and the transformation group of it is a subgroup of $\mathcal{T}$, hence is finite. Since $q$ is the order of $\mathcal{T}$ it follows that $T_{1}^{q}=T_{2}^{q}=I d_{\mathbb{R}^{d}}$. So taking the new IFS after relabeling we have that $T_{1}=T_{2}=I d_{\mathbb{R}^{d}}$. We can further assume that $r_{1}=r_{2}$ because if we iterate the IFS, we obtain the SS-IFS $\left\{S_{\mathbf{i}}: \mathbf{i} \in \mathcal{I}^{2}\right\}$ and again the similarity dimension, the attractor and the finiteness of the transformation group do not change. Then $r_{1} \cdot r_{2}=r_{2} \cdot r_{1}, T_{1} \circ T_{2}=I d_{\mathbb{R}^{d}} \circ I d_{\mathbb{R}^{d}}=T_{2} \circ T_{1}$. So taking the new IFS after relabeling we have that $T_{1}=T_{2}=I d_{\mathbb{R}^{d}}$ and $r_{1}=r_{2}$.

So $K_{1}=S_{1}(K)$ is a translate of $K_{2}=S_{2}(K)$. Let $v$ be the translation vector such that $K_{1}=K_{2}+v$. Let $M$ be the orthogonal direct complement of $v$ (if $v=0$ then $M \in G_{d, d-1}$ can be arbitrary). Then $\Pi_{M}\left(K_{1}\right)=\Pi_{M}\left(K_{2}\right)$. Let $L=\Pi_{M}: \mathbb{R}^{d} \longrightarrow \mathbb{R}^{d-1}$. Then let $G(\mathcal{V}, \mathcal{E})$ be the graph, $\left\{S_{e}: e \in \mathcal{E}\right\}$ be the GD-IFS that is constructed in the proof of Theorem 1.1 and for $i, j \in \mathcal{V}$ and for $n \in \mathcal{I}$, such that $O_{i} \circ T_{n}=O_{j}$, let $e_{i, j}^{n}$ as in the proof. Let $i \in \mathcal{V}$ 
such that $O_{i}=I d_{\mathbb{R}^{d}}$. Then $e_{i, i}^{1}$ and $e_{i, i}^{2}$ are loops in $G(\mathcal{V}, \mathcal{E})$ and

$$
\begin{aligned}
S_{e_{i, i}^{1}}\left(\Pi_{M}(K)\right) & =r_{1} \cdot \Pi_{M}(K)+\Pi_{M}\left(v_{1}\right)=r_{1} \cdot \Pi_{M}(K)+\Pi_{M}\left(v_{1}-v\right) \\
& =r_{2} \cdot \Pi_{M}(K)+\Pi_{M}\left(v_{2}\right)=S_{e_{i, i}^{2}}\left(\Pi_{M}(K)\right) .
\end{aligned}
$$

So if we take $\left\{S_{e}: e \in \mathcal{E} \backslash\left\{e_{0}\right\}\right\}$ with $e_{0}=e_{i, i}^{2}$ then $\left\{S_{e}: e \in \mathcal{E} \backslash\left\{e_{0}\right\}\right\}$ is a strongly connected GD-IFS with attractor $\left(\Pi_{M} \circ O_{1}(K), \ldots, \Pi_{M} \circ O_{q}(K)\right)$. So by Lemma 2.18 the similarity dimension of $\left\{S_{e}: e \in \mathcal{E} \backslash\left\{e_{0}\right\}\right\}$ is strictly smaller than $s$. Hence $\operatorname{dim}_{H}\left(\Pi_{M}(K)\right)<s$ by Lemma 2.11,

\section{Hausdorff measure of the orbits}

In this section we deal with the general results when we have no restriction on $\mathcal{T}$ and our main aim is to prove Theorem 1.3. At the end of this section we conclude Corollary 1.4 from Theorem 1.3.

First we prove Proposition 1.11 that says the Hausdorff measure of linear images of $K$ is upper semi-continuous in the linear maps. This observation is essential in the proof of Theorem 1.3 .

Proposition 5.1. Let $\left\{S_{i}\right\}_{i=1}^{m}$ be an $S S$-IFS with attractor $K \subseteq \mathbb{R}^{d}$, let $t=\operatorname{dim}_{H}(K)$ and $L: \mathbb{R}^{d} \longrightarrow \mathbb{R}^{d_{2}}$ be a linear map. Then for every $\varepsilon>0$ there exists $\delta>0$ such that for every linear map $L_{2}: \mathbb{R}^{d} \longrightarrow \mathbb{R}^{d_{2}}$ with $\left\|L-L_{2}\right\|<\delta$ we have that $\mathcal{H}^{t}\left(L_{2}(K)\right) \leq \mathcal{H}_{\infty}^{t}(L(K))+\varepsilon$.

Proof. It is enough to verify the proposition for $0<\varepsilon<1$. We may assume that $\mathcal{H}^{t}(K)>0$ otherwise $\mathcal{H}^{t}\left(L_{2}(K)\right)=0$. By Proposition 2.9 $\mathcal{H}^{t}(K)<\infty$ hence $K$ is a $t$-set. Since $K$ is compact there exists $R>0$ such that $K$ is contained in $B(0, R)$. Since $L(K)$ is compact it follows that $\mathcal{H}_{\infty}^{t}(L(K))<\infty$. Thus there exists a finite open cover $\mathcal{C}$ of $L(K)$ such that

$$
\sum_{C \in \mathcal{C}} \operatorname{diam}(C)^{t} \leq \mathcal{H}_{\infty}^{t}(L(K))+\varepsilon
$$

and $0<\operatorname{diam}(C)<\infty$. Let $d_{\max }=\max \{\operatorname{diam}(C): C \in \mathcal{C}\}$. Because $\mathcal{C}$ is a cover of $L(K)$ and $K \subseteq B(0, R)$ it follows that $K \subseteq \bigcup_{C \in \mathcal{C}} L^{-1}(C) \cap B(0, R)$ and $L^{-1}(C) \cap B(0, R)$ is a bounded set such that $\operatorname{diam}\left(L\left(L^{-1}(C) \cap B(0, R)\right)\right)=\operatorname{diam}(C)>0$ for each $C \in \mathcal{C}$. Hence for each $C \in \mathcal{C}$ we can find $\delta_{C}>0$ such that if $L_{2}: \mathbb{R}^{d} \longrightarrow \mathbb{R}^{d_{2}}$ is a linear map with $\left\|L-L_{2}\right\|<\delta_{C}$ then $\operatorname{diam}\left(L_{2}\left(L^{-1}(C) \cap B(0, R)\right)\right) \leq \operatorname{diam}(C) \cdot(1+\varepsilon)$. Let $\delta=$ $\min \left\{\delta_{C}: C \in \mathcal{C}\right\} /\|L\|+1>0$. So if $\left\|L-L_{2}\right\|<\delta$ for some linear map $L_{2}: \mathbb{R}^{d} \longrightarrow \mathbb{R}^{d_{2}}$ and $\left\|T-I d_{\mathbb{R}^{d}}\right\|<\delta$ for some $T \in \mathbb{O}_{d}$ then

$$
\left\|L-L_{2} \circ T\right\|=\left\|L-L \circ T+L \circ T-L_{2} \circ T\right\|<\delta(\|L\|+1)=\min \left\{\delta_{C}: C \in \mathcal{C}\right\} .
$$

Hence

$$
\operatorname{diam}\left(L_{2} \circ T\left(L^{-1}(C) \cap B(0, R)\right)\right) \leq \operatorname{diam}(C) \cdot(1+\varepsilon)<2 d_{\max } .
$$

The lemma will follow if we show that $\mathcal{H}_{\eta}^{t}\left(L_{2}(K)\right) \leq(1+\varepsilon)^{t} \cdot\left(\mathcal{H}_{\infty}^{t}(L(K))+\varepsilon\right)$ for every $\eta>0$ and linear map $L_{2}: \mathbb{R}^{d} \longrightarrow \mathbb{R}^{d_{2}}$ with $\left\|L-L_{2}\right\|<\delta$, where $\mathcal{H}_{\eta}^{t}$ denotes Hausdorff premeasure, used to define Hausdorff measure. Let $\eta>0$ be fixed, $r_{\max }=\max \left\{r_{i}: i \in \mathcal{I}\right\}$, let 
$k$ be a positive integer such that $r_{\max }^{k} \cdot 2 d_{\max }<\eta$ and $L_{2}: \mathbb{R}^{d} \longrightarrow \mathbb{R}^{d_{2}}$ be a linear map with $\left\|L-L_{2}\right\|<\delta$. Then $K$ is the attractor of the SS-IFS $\left\{S_{\mathbf{i}}: \mathbf{i} \in \mathcal{I}^{k}\right\}$. We apply Lemma 1.9] to the SS-IFS $\left\{S_{\mathbf{i}}: \mathbf{i} \in \mathcal{I}^{k}\right\}$ with $O=I d_{\mathbb{R}^{d}}$ and $\delta>0$ to obtain $\mathcal{I}_{\infty} \subseteq \bigcup_{k_{2}=1}^{\infty} \mathcal{I}^{k \cdot k_{2}}$ such that $\left\|T_{\mathbf{i}}-I d_{\mathbb{R}^{d}}\right\|<\delta$ for all $\mathbf{i} \in \mathcal{I}_{\infty}, K_{\mathbf{i}} \cap K_{\mathbf{j}}=\emptyset$ for $\mathbf{i}, \mathbf{j} \in \mathcal{I}_{\infty}, \mathbf{i} \neq \mathbf{j}$, and $\mathcal{H}^{t}\left(K \backslash\left(\bigcup_{\mathbf{i} \in \mathcal{I}_{\infty}} K_{\mathbf{i}}\right)\right)=0$. As $K$ is a $t$-set we have by Remark 1.10 that

$$
\sum_{\mathbf{i} \in \mathcal{I}_{\infty}} r_{\mathbf{i}}^{t}=1 .
$$

Since $\left\|T_{\mathbf{i}}-I d_{\mathbb{R}^{d}}\right\|<\delta$ for $\mathbf{i} \in \mathcal{I}_{\infty}$ and $\left\|L-L_{2}\right\|<\delta$, it follows from (13) that

$$
\begin{aligned}
\operatorname{diam}\left(L_{2}\left(S_{\mathbf{i}}\left(L^{-1}(C) \cap B(0, R)\right)\right)\right) & \leq r_{\mathbf{i}} \cdot \operatorname{diam}\left(L_{2}\left(T_{\mathbf{i}}\left(L^{-1}(C) \cap B(0, R)\right)\right)\right) \\
& \leq r_{\mathbf{i}} \cdot \operatorname{diam}(C) \cdot(1+\varepsilon)<r_{\mathbf{i}} \cdot 2 d_{\max } \leq r_{\max }^{k} \cdot 2 d_{\max }<\eta
\end{aligned}
$$

Thus $\left\{L_{2}\left(S_{\mathbf{i}}\left(L^{-1}(C) \cap B(0, R)\right)\right): C \in \mathcal{C}, \mathbf{i} \in \mathcal{I}_{\infty}\right\}$ is an $\eta$-cover of $L_{2}\left(\bigcup_{\mathbf{i} \in \mathcal{I}_{\infty}} K_{\mathbf{i}}\right)$ and

$$
\begin{aligned}
\mathcal{H}_{\eta}^{t}\left(L_{2}\left(\bigcup_{\mathbf{i} \in \mathcal{I}_{\infty}} K_{\mathbf{i}}\right)\right) & \leq \sum_{C \in \mathcal{C}} \sum_{\mathbf{i} \in \mathcal{I}_{\infty}} \operatorname{diam}\left(L_{2}\left(S_{\mathbf{i}}\left(L^{-1}(C) \cap B(0, R)\right)\right)\right)^{t} \\
& \leq \sum_{C \in \mathcal{C}} \sum_{\mathbf{i} \in \mathcal{I}_{\infty}}\left(r_{\mathbf{i}} \cdot \operatorname{diam}(C) \cdot(1+\varepsilon)\right)^{t} \\
& \leq(1+\varepsilon)^{t} \sum_{C \in \mathcal{C}} \operatorname{diam}(C)^{t} \sum_{\mathbf{i} \in \mathcal{I}_{\infty}} r_{\mathbf{i}}^{t} \leq(1+\varepsilon)^{t} \sum_{C \in \mathcal{C}} \operatorname{diam}(C)^{t} \\
& \leq(1+\varepsilon)^{t} \cdot\left(\mathcal{H}_{\infty}^{t}(L(K))+\varepsilon\right)
\end{aligned}
$$

where we used (12), (14) and (15).

Since $\mathcal{H}^{t}\left(K \backslash\left(\bigcup_{\mathbf{i} \in \mathcal{I}_{\infty}} K_{\mathbf{i}}\right)\right)=0$ it follows $\mathcal{H}_{\eta}^{t}\left(L_{2}\left(K \backslash\left(\bigcup_{\mathbf{i} \in \mathcal{I}_{\infty}} K_{\mathbf{i}}\right)\right)\right)=0$. Thus by (16)

$$
\mathcal{H}_{\eta}^{t}\left(L_{2}(K)\right) \leq(1+\varepsilon)^{t} \cdot\left(\mathcal{H}_{\infty}^{t}(L(K))+\varepsilon\right)
$$

which completes the proof.

Proof of Proposition 1.11. The proposition is an immediate consequence of Proposition 5.1, $\square$

Proof of Theorem 1.3. By Proposition 2.9 $\mathcal{H}^{t}(K)<\infty$ hence $K$ is a $t$-set. Let $\varepsilon>0$ be arbitrary. Let $\delta>0$ such that for every linear map $L_{2}: \mathbb{R}^{d} \longrightarrow \mathbb{R}^{d_{2}}$ with $\left\|L-L_{2}\right\|<\delta$ we have that $\mathcal{H}^{t}\left(L_{2}(K)\right) \leq \mathcal{H}^{t}(L(K))+\varepsilon$. Such a $\delta>0$ exists by Proposition 1.11, Let $\mathcal{I}_{\infty}$ be the set provided by Proposition 1.9 for $O^{-1}$ in place of $O$ and $\frac{\delta}{\|L\|}$ in place of $\delta$. Then $\left\|O \circ T_{\mathbf{i}}-I d_{\mathbb{R}^{d}}\right\|=\left\|T_{\mathbf{i}}-O^{-1}\right\|<\frac{\delta}{\|L\|}$ for every $\mathbf{i} \in \mathcal{I}_{\infty}$, hence $\left\|L \circ O \circ T_{\mathbf{i}}-L\right\| \leq$ $\|L\| \cdot\left\|O \circ T_{\mathbf{i}}-I d_{\mathbb{R}^{d}}\right\|<\delta$. So $\mathcal{H}^{t}\left(L \circ O \circ T_{\mathbf{i}}(K)\right) \leq \mathcal{H}^{t}(L(K))+\varepsilon$ for every $\mathbf{i} \in \mathcal{I}_{\infty}$, hence

$$
\mathcal{H}^{t}\left(L \circ O\left(S_{\mathbf{i}}(K)\right)\right)=r_{\mathbf{i}}^{t} \cdot \mathcal{H}^{t}\left(L \circ O \circ T_{\mathbf{i}}(K)\right) \leq r_{\mathbf{i}}^{t} \cdot\left(\mathcal{H}^{t}(L(K))+\varepsilon\right) .
$$

Since $K$ is a $t$-set we have by Remark 1.10 that

$$
\sum_{\mathbf{i} \in \mathcal{I}_{\infty}} r_{\mathbf{i}}^{t}=1 .
$$


It follows that

$$
\sum_{\mathbf{i} \in \mathcal{I}_{\infty}} \mathcal{H}^{t}\left(L \circ O\left(S_{\mathbf{i}}(K)\right)\right) \leq \sum_{\mathbf{i} \in \mathcal{I}_{\infty}} r_{\mathbf{i}}^{t} \cdot\left(\mathcal{H}^{t}(L(K))+\varepsilon\right)=\mathcal{H}^{t}(L(K))+\varepsilon
$$

where we have used (17) and (18). Because $\mathcal{H}^{t}\left(L \circ O\left(K \backslash\left(\bigcup_{\mathbf{i} \in \mathcal{I}_{\infty}} S_{\mathbf{i}}(K)\right)\right)\right)=0$ it follows that $\mathcal{H}^{t}(L \circ O(K)) \leq \mathcal{H}^{t}(L(K))+\varepsilon$ for all $\varepsilon>0$. Hence $\mathcal{H}^{t}(L \circ O(K)) \leq \mathcal{H}^{t}(L(K))$. Replacing $L$ by $L \circ O$ and $O$ by $O^{-1}$, with the same argument we get that $\mathcal{H}^{t}(L(K))=$ $\mathcal{H}^{t}\left(L \circ O \circ O^{-1}(K)\right) \leq \mathcal{H}^{t}(L \circ O(K))$. Thus $\mathcal{H}^{t}(L \circ O(K))=\mathcal{H}^{t}(L(K))$ and so (7) holds for $A=K$.

Let $\mathbf{i} \in \mathcal{I}^{k}$ for some $k \in \mathbb{N}$. Then

$$
\begin{aligned}
\mathcal{H}^{t}\left(L \circ O\left(K_{\mathbf{i}}\right)\right) & =\mathcal{H}^{t}\left(L \circ O\left(S_{\mathbf{i}}(K)\right)\right)=\mathcal{H}^{t}\left(L \circ O\left(r_{\mathbf{i}} \cdot T_{\mathbf{i}}(K)+v_{\mathbf{i}}\right)\right) \\
& =r_{\mathbf{i}}^{t} \cdot \mathcal{H}^{t}\left(L \circ O \circ T_{\mathbf{i}}(K)\right)=\frac{\mathcal{H}^{t}\left(K_{\mathbf{i}}\right)}{\mathcal{H}^{t}(K)} \cdot \mathcal{H}^{t}(L(K))
\end{aligned}
$$

where we used (7) when $A=K$. So (17) holds for $A=K_{\mathbf{i}}$, for each $\mathbf{i} \in \mathcal{I}^{k}, k \in \mathbb{N}$.

Let $\mathcal{J}$ be the set provided by Lemma 3.1. For every $k \in \mathbb{N}$ let us denote the set $\left\{\mathbf{i}_{1} * \ldots * \mathbf{i}_{k}: \mathbf{i}_{1}, \ldots, \mathbf{i}_{k} \in \mathcal{J}\right\}$ by $\mathcal{J}^{k}$. For $k \in \mathbb{N}$

$$
\mathcal{H}^{t}\left(K \backslash\left(\bigcup_{\mathbf{i} \in \mathcal{J}^{k}} K_{\mathbf{i}}\right)\right)=0
$$

thus

$$
\mathcal{H}^{t}(K)=\mathcal{H}^{t}\left(\bigcup_{\mathbf{i} \in \mathcal{J}^{k}} K_{\mathbf{i}}\right)=\sum_{\mathbf{i} \in \mathcal{J}^{k}} \mathcal{H}^{t}\left(K_{\mathbf{i}}\right)
$$

So

$$
\begin{aligned}
\sum_{\mathbf{i} \in \mathcal{J}^{k}} \mathcal{H}^{t}\left(L \circ O\left(K_{\mathbf{i}}\right)\right) & =\sum_{\mathbf{i} \in \mathcal{J}^{k}} \frac{\mathcal{H}^{t}\left(K_{\mathbf{i}}\right)}{\mathcal{H}^{t}(K)} \cdot \mathcal{H}^{t}(L(K))=\frac{\mathcal{H}^{t}(K)}{\mathcal{H}^{t}(K)} \cdot \mathcal{H}^{t}(L(K)) \\
& =\mathcal{H}^{t}(L \circ O(K))=\mathcal{H}^{t}\left(L \circ O\left(\bigcup_{\mathbf{i} \in \mathcal{J}^{k}} K_{\mathbf{i}}\right)\right)
\end{aligned}
$$

where we used (7) for $A=K_{\mathbf{i}}$ and for $A=K$. It follows that $\mathcal{H}^{t}\left(L \circ O\left(K_{\mathbf{i}}\right) \cap L \circ O\left(K_{\mathbf{j}}\right)\right)=0$ for $\mathbf{i}, \mathbf{j} \in \mathcal{J}^{k}, \mathbf{i} \neq \mathbf{j}$. Hence (17) holds for $A=\bigcup_{\mathbf{i} \in \mathcal{F}} K_{\mathbf{i}}$ where $\mathcal{F} \subseteq \mathcal{J}^{k}$.

Using (19) and the continuity of measures it follows that

$$
\mathcal{H}^{t}\left(K \backslash\left(\bigcap_{k=1}^{\infty} \bigcup_{\mathbf{i} \in \mathcal{J}^{k}} K_{\mathbf{i}}\right)\right)=0
$$

Assume that $A \subseteq \bigcap_{k=1}^{\infty} \bigcup_{\mathbf{i} \in \mathcal{J}^{k}} K_{\mathbf{i}}$ is compact, $\varepsilon>0$ arbitrary and let

$$
F_{k}=\bigcup_{\mathbf{i} \in \mathcal{J}^{k}, K_{\mathbf{i}} \cap A \neq \emptyset} K_{\mathbf{i}}
$$


Then $K \supseteq F_{1} \supseteq F_{2} \supseteq \ldots$ and $A=\bigcap_{k=1}^{\infty} F_{k}$. Thus there exists $k$ such that $\mathcal{H}^{t}\left(F_{k} \backslash A\right)<\varepsilon$. Since (17) holds for $F_{k}$ it follows that

$$
\begin{aligned}
\mathcal{H}^{t}(L \circ O(A)) & \leq \mathcal{H}^{t}\left(L \circ O\left(F_{k}\right)\right)=\frac{\mathcal{H}^{t}\left(F_{k}\right)}{\mathcal{H}^{t}(K)} \mathcal{H}^{t}(L(K)) \\
& =\frac{\mathcal{H}^{t}(A)+\mathcal{H}^{t}\left(F_{k} \backslash A\right)}{\mathcal{H}^{t}(K)} \mathcal{H}^{t}(L(K)) \leq \frac{\mathcal{H}^{t}(A)+\varepsilon}{\mathcal{H}^{t}(K)} \mathcal{H}^{t}(L(K))
\end{aligned}
$$

and

$$
\begin{aligned}
\mathcal{H}^{t}(L \circ O(A)) & \geq \mathcal{H}^{t}\left(L \circ O\left(F_{k}\right)\right)-\mathcal{H}^{t}\left(L \circ O\left(F_{k} \backslash A\right)\right) \\
& \geq \frac{\mathcal{H}^{t}\left(F_{k}\right)}{\mathcal{H}^{t}(K)} \mathcal{H}^{t}(L(K))-\|L \circ O\|^{t} \cdot \mathcal{H}^{t}\left(F_{k} \backslash A\right) \\
& \geq \frac{\mathcal{H}^{t}(A)}{\mathcal{H}^{t}(K)} \mathcal{H}^{t}(L(K))-\|L\|^{t} \cdot \varepsilon .
\end{aligned}
$$

Since $\varepsilon>0$ is arbitrary (7) holds for compact $A \subseteq \bigcap_{k=1}^{\infty} \bigcup_{\mathbf{i} \in \mathcal{J}^{k}} K_{\mathbf{i}}$.

Now assume that $A$ is any $\mathcal{H}^{t}$-measurable set and $\varepsilon>0$ is arbitrary. By (20)

$$
\mathcal{H}^{t}\left(A \bigcap\left(\bigcap_{k=1}^{\infty} \bigcup_{\mathbf{i} \in \mathcal{J}^{k}} K_{\mathrm{i}}\right)\right)=\mathcal{H}^{t}(A) .
$$

Hence we can find a compact $F \subseteq A \bigcap\left(\bigcap_{k=1}^{\infty} \bigcup_{\mathbf{i} \in \mathcal{J}^{k}} K_{\mathbf{i}}\right) \subseteq A$ such that $\mathcal{H}^{t}(A \backslash F)<\varepsilon$. Using a similar argument to (211) and (221) we can deduce that $\mathcal{H}^{t}(L \circ O(A))=\frac{\mathcal{H}^{t}(A)}{\mathcal{H}^{t}(K)} \mathcal{H}^{t}(L(K))$ because (77) holds for $F$. Thus (17) holds for every $\mathcal{H}^{t}$-measurable $A$.

Now let $A \subseteq K$ be arbitrary and let $B$ be a $\mathcal{H}^{t}$-measurable hull of $A$ such that $A \subseteq B \subseteq K$. By virtue of Lemma 2.5 and applying (77) to $B$ we get that (77) holds for $A$.

Proof of Corollary 1.4. If $\mathcal{H}^{t}(K)=0$ then the statement is trivial, so we can assume that $\mathcal{H}^{t}(K)>0$. Since $B \subseteq(K \backslash A) \cup(A \cap B)$ and $\mathcal{H}^{t}(A \cap B)=0$ it is enough to show that $\mathcal{H}^{t}(L(A) \cap L(K \backslash A))=0$. By Theorem 1.3

$$
\begin{aligned}
\mathcal{H}^{t}(L(K)) & =\mathcal{H}^{t}(L(A) \cup L(K \backslash A)) \\
& =\mathcal{H}^{t}(L(A))+\mathcal{H}^{t}(L(K \backslash A))-\mathcal{H}^{t}(L(A) \cap L(K \backslash A)) \\
& =\frac{\mathcal{H}^{t}(A)}{\mathcal{H}^{t}(K)} \mathcal{H}^{t}(L(K))+\frac{\mathcal{H}^{t}(K \backslash A)}{\mathcal{H}^{t}(K)} \mathcal{H}^{t}(L(K))-\mathcal{H}^{t}(L(A) \cap L(K \backslash A)) \\
& =\mathcal{H}^{t}(L(K))-\mathcal{H}^{t}(L(A) \cap L(K \backslash A)) .
\end{aligned}
$$

Hence $\mathcal{H}^{t}(L(A) \cap L(K \backslash A))=0$ since $\mathcal{H}^{t}(L(K))<\infty$ by Proposition 2.9,

\section{Transformation groups of dense orbits and Hausdorff measure}

In this section our main goal is to prove Theorem 1.5. First we show that under the assumptions of Theorem 1.5 every linear image of $K$ is of zero measure. Then we generalise this for continuously differentiable maps. 
Lemma 6.1. Let $\mathcal{G}$ be a closed subset of $\mathbb{O}_{d}$, let $K \subseteq \mathbb{R}^{d}$ be a compact set, let $L: \mathbb{R}^{d} \longrightarrow \mathbb{R}^{d_{2}}$ be a linear map and $c>0$ be such that $\mathcal{H}_{\infty}^{t}(L \circ O(K))<c$ for every $O \in \mathcal{G}$. Then there exists $\zeta>0$ such that for every $O \in \mathcal{G}$ there exists a finite open cover $\mathcal{U}$ of $L \circ O(K)$ such that $\sum_{U \in \mathcal{U}} \operatorname{diam}(U)^{t}<c$ and $\min _{U \in \mathcal{U}} \operatorname{diam}(U)>\zeta$.

Proof. For every $O \in \mathcal{G}$ we can find a finite open cover $\mathcal{U}_{O}$ of $L \circ O(K)$ and $0<\varepsilon_{O}<\frac{1}{2}$ such that $\sum_{U \in \mathcal{U}_{O}} \operatorname{diam}(U)^{t} \cdot\left(1+2 \varepsilon_{O}\right)^{t}<c$. Let $\zeta_{O}=\min _{U \in \mathcal{U}_{O}} \operatorname{diam}(U)>0$ and $\widehat{U}$ be the $\zeta_{O} \cdot \varepsilon_{O}$-neigbourhood of $U$ for each $U \in \mathcal{U}_{O}$. We can find $\delta_{O}>0$ such that if $O_{2} \in \mathbb{O}_{d}$ and $\left\|O-O_{2}\right\|<\delta_{O}$ then $L \circ O_{2}(K)$ is contained in the $\zeta_{O} \cdot \varepsilon_{O}$-neighbourhood of $L \circ O(K)$, hence $L \circ O_{2}(K)$ is covered by $\left\{\widehat{U}: U \in \mathcal{U}_{O}\right\}$. Then $\left\{\widehat{U}: U \in \mathcal{U}_{O}\right\}$ is an open cover of $L \circ O_{2}(K)$,

$$
\sum_{U \in \mathcal{U}_{O}} \operatorname{diam}(\widehat{U})^{t} \leq \sum_{U \in \mathcal{U}_{O}} \operatorname{diam}(U)^{t} \cdot\left(1+2 \varepsilon_{O}\right)^{t}<c
$$

and $\min _{U \in \mathcal{U}_{O}} \operatorname{diam}(\widehat{U})>\zeta_{O}$.

As $\mathcal{G}$ is compact, we can find finitely many orthogonal transformations $O_{1}, \ldots, O_{n} \in \mathcal{G}$ such that for every $O \in \mathcal{G}$ there exists $i \in\{1, \ldots, n\}$ with $\left\|O_{i}-O\right\|<\delta_{O_{i}}$. Hence $\zeta=$ $\min _{1 \leq i \leq n} \zeta_{O_{i}}$ satisfies the statement.

For $r \in \mathbb{R}, r>0$ and $H \subseteq \mathbb{R}^{d}$ we denote the $r$-neigbourhood of $H$ by $B(H, r)$, i.e. $B(H, r)=\left\{x \in \mathbb{R}^{d}: \exists y \in H,\|x-y\|<r\right\}$.

Proposition 6.2. Let $\left\{S_{i}\right\}_{i=1}^{m}$ be an $S S$-IFS with attractor $K, t=\operatorname{dim}_{H}(K)$ and $L: \mathbb{R}^{d} \longrightarrow$ $\mathbb{R}^{d_{2}}$ be a linear map with $\operatorname{rank}(L)=l$. If $1 \leq l<d$ and there exists $M \in G_{d, l}$ such that the set $\{O(M): O \in \mathcal{T}\}$ is dense in $G_{d, l}$ then $\mathcal{H}^{t}(L(K))=0$.

We first show that there exist two words $a$ and $b$ and $O_{0} \in \mathbb{O}_{d}$ such that $L \circ O_{0}\left(K_{a}\right)$ and $L \circ O_{0}\left(K_{b}\right)$ have very large overlap. Then we use a variant of Proposition 1.9 to show that, due to self-similarity, this remains valid at all scales. Finally we conclude that due to these overlaps the measure must collapse.

Proof. It holds in general that $\mathcal{H}^{t}(H)=0$ if and only if $\mathcal{H}_{\infty}^{t}(H)=0$. Hence it is enough to show that $\mathcal{H}_{\infty}^{t}(L(K))=0$. We can assume that $\mathcal{H}^{t}(K)>0$ otherwise the statement is trivial. By Proposition 2.9 $\mathcal{H}^{t}(K)<\infty$, hence $K$ is a $t$-set. It follows that $\mathcal{H}^{t}(L(K))<\infty$ and by Theorem 1.3 and Proposition $1.11 \mathcal{H}_{\infty}^{t}(L(K))=\mathcal{H}^{t}(L(K))=\mathcal{H}^{t}\left(L \circ O_{0}(K)\right)=$ $\mathcal{H}_{\infty}^{t}\left(L \circ O_{0}(K)\right)$ for every $O_{0} \in \overline{\mathcal{T}}$. Let $\varepsilon>0$ be arbitrary, $\mathcal{G}=\overline{\mathcal{T}}, c=\mathcal{H}_{\infty}^{t}(L(K))+\varepsilon$ and $\zeta>0$ be the $\zeta$ provided by Lemma 6.1. We can find $\delta>0$ such that for every linear map $L_{2}: \mathbb{R}^{d} \longrightarrow \mathbb{R}^{d}$ such that $\left\|I d_{\mathbb{R}^{d}}-L_{2}\right\|<\delta$ we have that $L_{2}(K) \subseteq B(K, \varepsilon \zeta)$. By Proposition 1.9 we can find $\mathbf{i}_{1}, \mathbf{i}_{2} \in \bigcup_{k=1}^{\infty} \mathcal{I}^{k}$ such that $K_{\mathbf{i}_{1}} \cap K_{\mathbf{i}_{2}}=\emptyset$ and $\left\|T_{\mathbf{i}_{1}}-I d_{\mathbb{R}^{d}}\right\|<\frac{\delta}{4}$, $\left\|T_{\mathbf{i}_{2}}-I d_{\mathbb{R}^{d}}\right\|<\frac{\delta}{4}$. Let $a=\mathbf{i}_{1} * \mathbf{i}_{2}$ and $b=\mathbf{i}_{2} * \mathbf{i}_{1}$. Then $\left\|T_{a}-I d_{\mathbb{R}^{d}}\right\|<\frac{\delta}{2},\left\|T_{b}-I d_{\mathbb{R}^{d}}\right\|<\frac{\delta}{2}$, $K_{a} \cap K_{b}=\emptyset$ and $r_{a}=r_{b}$. Let $v=S_{b}(0)-S_{a}(0)$ and $O_{0} \in \overline{\mathcal{T}}$ such that $L \circ O_{0}(v)=0$. We can choose such an $O_{0}$ by Lemma 2.6. We can find $\delta_{2}>0$ such that if $\left\|L \circ O_{0}-L_{2}\right\|<\delta_{2}$ then $\left\|L_{2}(v)\right\|<r_{a} \varepsilon \zeta$.

We can apply Proposition 3.6 with $\min \left\{\frac{\delta}{2}, \frac{\delta_{2}}{\|L\|}\right\}$ replacing $\delta, a$ replacing $\mathbf{i}_{1}, b$ replacing $\mathbf{i}_{2}, n=2$ and $O=I d_{\mathbb{R}^{d}}$ to obtain $\mathcal{I}_{\infty} \subseteq \bigcup_{k=1}^{\infty} \mathcal{I}^{k}$ such that $\left\|T_{\mathbf{i}}-I d_{\mathbb{R}^{d}}\right\|<\min \left\{\frac{\delta}{2}, \frac{\delta_{2}}{\|L\|}\right\}$ for all $\mathbf{i} \in \mathcal{I}_{\infty}$, with $\bigcup_{\mathbf{i} \in \mathcal{I}_{\infty}}\left(K_{\mathbf{i} * a} \cup K_{\mathbf{i} * b}\right)$ a disjoint union and $\mathcal{H}^{t}\left(K \backslash\left(\bigcup_{\mathbf{i} \in \mathcal{I}_{\infty}}\left(K_{\mathbf{i} * a} \cup K_{\mathbf{i} * b}\right)\right)\right)=0$. 
So $\left\|T_{\mathbf{i}} \circ T_{a}-I d_{\mathbb{R}^{d}}\right\|<\delta$ and $\left\|T_{\mathbf{i}} \circ T_{b}-I d_{\mathbb{R}^{d}}\right\|<\delta$, hence $T_{\mathbf{i}} \circ T_{a}(K) \subseteq B(K, \varepsilon \zeta)$ and $T_{\mathbf{i}} \circ T_{b}(K) \subseteq B(K, \varepsilon \zeta)$. Thus

$$
r_{\mathbf{i}} r_{a} T_{\mathbf{i}} \circ T_{a}(K) \cup r_{\mathbf{i}} r_{b} T_{\mathbf{i}} \circ T_{b}(K) \subseteq B\left(r_{\mathbf{i}} r_{a} K, r_{\mathbf{i}} r_{a} \varepsilon \zeta\right)
$$

since $r_{a}=r_{b}$. Hence

$$
O_{0} \circ S_{\mathbf{i} * a}(K) \subseteq B\left(r_{\mathbf{i}} r_{a} O_{0}(K)+O_{0} \circ S_{\mathbf{i} * a}(0), r_{\mathbf{i}} r_{a} \varepsilon \zeta\right)
$$

and

$$
O_{0} \circ S_{\mathbf{i} * b}(K) \subseteq B\left(r_{\mathbf{i}} r_{a} O_{0}(K)+O_{0} \circ S_{\mathbf{i} * b}(0), r_{\mathbf{i}} r_{a} \varepsilon \zeta\right)
$$

Hence

$$
L \circ O_{0} \circ S_{\mathbf{i} * a}(K) \subseteq B\left(r_{\mathbf{i}} r_{a} L \circ O_{0}(K)+L \circ O_{0} \circ S_{\mathbf{i} * a}(0),\|L\| r_{\mathbf{i}} r_{a} \varepsilon \zeta\right)
$$

and

$$
L \circ O_{0} \circ S_{\mathbf{i} * b}(K) \subseteq B\left(r_{\mathbf{i}} r_{a} L \circ O_{0}(K)+L \circ O_{0} \circ S_{\mathbf{i} * a}(0)+L \circ O_{0} \circ r_{\mathbf{i}} T_{\mathbf{i}}(v),\|L\| r_{\mathbf{i}} r_{a} \varepsilon \zeta\right) .
$$

By the choice of $\delta_{2}$ we have $\left\|L \circ O_{0} \circ r_{\mathbf{i}} T_{\mathbf{i}}(v)\right\|<r_{\mathbf{i}} r_{a} \varepsilon \zeta$. Hence $L \circ O_{0} \circ S_{\mathbf{i} * a}(K) \cup L \circ O_{0} \circ S_{\mathbf{i} * b}(K) \subseteq B\left(r_{\mathbf{i}} r_{a} L \circ O_{0}(K)+L \circ O_{0} \circ S_{\mathbf{i} * a}(0),(\|L\|+1) r_{\mathbf{i}} r_{a} \varepsilon \zeta\right)$.

By the choice of $\zeta$ there exists an open cover $\mathcal{U}$ of $L \circ O_{0}(K)$ such that $\sum_{U \in \mathcal{U}} \operatorname{diam}(U)^{t}<$ $\mathcal{H}_{\infty}^{t}(L(K))+\varepsilon$ and $\min _{U \in \mathcal{U}} \operatorname{diam}(U)>\zeta$. Let $\widehat{U}=B(U,(\|L\|+1) \varepsilon \zeta)$ for each $U \in$ $\mathcal{U}$ and $\mathcal{A}=\left\{r_{\mathbf{i}} r_{a} \widehat{U}+L \circ O_{0} \circ S_{\mathbf{i} * a}(0): U \in \mathcal{U}, \mathbf{i} \in \mathcal{I}_{\infty}\right\}$. Then $\mathcal{A}$ is an open cover of $L \circ$ $O_{0}\left(\bigcup_{\mathbf{i} \in \mathcal{I}_{\infty}}\left(K_{\mathbf{i} * a} \cup K_{\mathbf{i} * b}\right)\right)$.

Because $K$ is a $t$-set it follows in a similar way to Remark 1.10 that $\sum_{\mathbf{i} \in \mathcal{I}_{\infty}} r_{\mathbf{i}}^{t}\left(r_{a}^{t}+r_{b}^{t}\right)=1$, hence $\sum_{\mathbf{i} \in \mathcal{I}_{\infty}} r_{\mathbf{i}}^{t}\left(r_{a}^{t}\right)=\frac{1}{2}$ because $r_{a}=r_{b}$. Thus

$$
\begin{aligned}
\sum_{A \in \mathcal{A}} \operatorname{diam}(A)^{t} & \leq \sum_{U \in \mathcal{U}} \sum_{\mathbf{i} \in \mathcal{I}_{\infty}} r_{\mathbf{i}}^{t} r_{a}^{t}(\operatorname{diam}(U)+2(\|L\|+1) \varepsilon \zeta)^{t} \\
& \leq \sum_{U \in \mathcal{U}} \sum_{\mathbf{i} \in \mathcal{I}_{\infty}} r_{\mathbf{i}}^{t} r_{a}^{t} \operatorname{diam}(U)^{t}(1+2(\|L\|+1) \varepsilon)^{t} \\
& =\sum_{U \in \mathcal{U}} \frac{1}{2} \operatorname{diam}(U)^{t}(1+2(\|L\|+1) \varepsilon)^{t} \\
& \leq \frac{1}{2}\left(\mathcal{H}_{\infty}^{t}(L(K))+\varepsilon\right)(1+2(\|L\|+1) \varepsilon)^{t}
\end{aligned}
$$

Because $\mathcal{H}^{t}\left(K \backslash\left(\bigcup_{\mathbf{i} \in \mathcal{I}_{\infty}}\left(K_{\mathbf{i} * a} \cup K_{\mathbf{i} * b}\right)\right)\right)=0$ it follows that

$$
\mathcal{H}_{\infty}^{t}\left(L \circ O_{0}\left(K \backslash\left(\bigcup_{\mathbf{i} \in \mathcal{I}_{\infty}}\left(K_{\mathbf{i} * a} \cup K_{\mathbf{i} * b}\right)\right)\right)\right)=0 .
$$

Hence

$$
\mathcal{H}_{\infty}^{t}\left(L \circ O_{0}(K)\right) \leq \frac{1}{2}\left(\mathcal{H}_{\infty}^{t}(L(K))+\varepsilon\right)(1+2(\|L\|+1) \varepsilon)^{t} .
$$

Since this is true for all $\varepsilon>0$ it follows that $\mathcal{H}_{\infty}^{t}(L(K))=\mathcal{H}_{\infty}^{t}\left(L \circ O_{0}(K)\right) \leq \frac{1}{2} \cdot \mathcal{H}_{\infty}^{t}(L(K))$. Thus $\mathcal{H}_{\infty}^{t}(L(K))=0$. 
Corollary 6.3. Let $\left\{S_{i}\right\}_{i=1}^{m}$ be an SS-IFS with attractor $K$, let $t=\operatorname{dim}_{H}(K)$ and $L: \mathbb{R}^{d} \longrightarrow$ $\mathbb{R}^{d_{2}}$ be a linear map with $\operatorname{rank}(L) \leq l$. If $1 \leq l<d$ and there exists $M \in G_{d, l}$ such that the set $\{O(M): O \in \mathcal{T}\}$ is dense in $G_{d, l}$ then $\mathcal{H}^{t}(L(K))=0$.

Proof. If $L: \mathbb{R}^{d} \longrightarrow \mathbb{R}^{d_{2}}$ is a linear map of rank $k$ and $k \leq l<d$ then $\operatorname{dim} \operatorname{Ker}(L)=d-k$. Let $N \in G_{d, d-l}$ such that $N \subseteq \operatorname{Ker}(L)$. Then $L=L \circ \Pi_{N^{\perp}}$. It follows from Proposition 6.2 that $\mathcal{H}^{t}\left(\Pi_{N^{\perp}}(K)\right)=0$. Hence $\mathcal{H}^{t}(L(K))=0$.

Lemma 6.4. Let $K \subseteq \mathbb{R}^{d}$ be a compact set and c, $M>0$ be constants such that $\mathcal{H}_{\infty}^{t}(L(K))<$ c for every linear map $L: \mathbb{R}^{d} \longrightarrow \mathbb{R}^{d_{2}}$ with $\|L\| \leq M$. Then there exists $\zeta>0$ such that for every linear map $L: \mathbb{R}^{d} \longrightarrow \mathbb{R}^{d_{2}}$ with $\|L\| \leq M$ there exists a finite open cover $\mathcal{U}$ of $L(K)$ such that $\sum_{U \in \mathcal{U}} \operatorname{diam}(U)^{t}<c$ and $\min _{U \in \mathcal{U}} \operatorname{diam}(U)>\zeta$.

Lemma 6.4 can be proven similarly to Lemma 6.1 due the fact that the unit ball of the set of linear maps between two finite dimensional Euclidean spaces is compact.

Proof of Theorem 1.5. We can assume that $\mathcal{H}^{t}(K)>0$ otherwise the statement is trivial since $g$ is a Lipschitz map. By Proposition $2.9 \mathcal{H}^{t}(K)<\infty$ and hence $K$ is a $t$-set. Let $\varepsilon>0$ be fixed.

Let $x_{0} \in K$ be arbitrary. It follows from Corollary 6.3 that

$$
\mathcal{H}_{\infty}^{t}\left(g^{\prime}\left(S_{\mathbf{i}}\left(x_{0}\right)\right) \circ T_{\mathbf{i}}(K)\right)=\mathcal{H}^{t}\left(g^{\prime}\left(S_{\mathbf{i}}\left(x_{0}\right)\right) \circ T_{\mathbf{i}}(K)\right)=0 .
$$

Let $c=\varepsilon$ and $M=\sup \left\{\left\|g^{\prime}(x)\right\|: x \in K\right\}<\infty$, then let $\zeta>0$ be the $\zeta$ provided by Lemma 6.4. Hence for every $\mathbf{i} \in \bigcup_{k=1}^{\infty} \mathcal{I}^{k}$ there exists a finite open cover $\mathcal{U}_{\mathbf{i}}$ of $g^{\prime}\left(S_{\mathbf{i}}\left(x_{0}\right)\right) \circ T_{\mathbf{i}}(K)$ such that

$$
\sum_{U \in \mathcal{U}_{\mathbf{i}}} \operatorname{diam}(U)^{t}<\varepsilon
$$

and $\min _{U \in \mathcal{U}_{\mathrm{i}}} \operatorname{diam}(U)>\zeta$.

From the continuous differentiability of $g$ and the compactness of $K$ it follows that we can find $\delta>0$ such that

$$
\left\|g(y)-g(x)-g^{\prime}(x) \cdot(y-x)\right\|<\zeta \cdot\|y-x\|
$$

for $x, y \in K$ such that $\|y-x\|<\delta$ (see [4, Exercise 7(c).3]). By Lemma 3.1 and Remark 3.2 we can find $\mathcal{J} \subseteq \bigcup_{k=1}^{\infty} \mathcal{I}^{k}$ such that $K_{\mathbf{i}} \cap K_{\mathbf{j}}=\emptyset$ for $\mathbf{i}, \mathbf{j} \in \mathcal{J}, \mathbf{i} \neq \mathbf{j}$ and $\operatorname{diam}\left(K_{\mathbf{i}}\right)<\delta$ for every $\mathbf{i} \in \mathcal{J}$ and

$$
\mathcal{H}^{t}\left(K \backslash\left(\bigcup_{\mathbf{i} \in \mathcal{J}} K_{\mathbf{i}}\right)\right)=0 .
$$

Similarly to Remark 1.10 it follows that

$$
\sum_{\mathbf{i} \in \mathcal{J}} r_{\mathbf{i}}^{t}=1 .
$$

For every $y \in K$ we have that $\left\|S_{\mathbf{i}}(y)-S_{\mathbf{i}}\left(x_{0}\right)\right\| \leq \operatorname{diam}\left(K_{\mathbf{i}}\right)<\delta$ and hence by the choice of $\delta$ it follows that

$\left\|g\left(S_{\mathbf{i}}(y)\right)-g\left(S_{\mathbf{i}}\left(x_{0}\right)\right)-g^{\prime}\left(S_{\mathbf{i}}\left(x_{0}\right)\right) \cdot\left(S_{\mathbf{i}}(y)-S_{\mathbf{i}}\left(x_{0}\right)\right)\right\|<\zeta \cdot\left\|S_{\mathbf{i}}(y)-S_{\mathbf{i}}\left(x_{0}\right)\right\| \leq \zeta r_{\mathbf{i}} \operatorname{diam}(K)$. 
Thus

$$
g\left(S_{\mathbf{i}}(y)\right) \in B\left(g^{\prime}\left(S_{\mathbf{i}}\left(x_{0}\right)\right)\left(S_{\mathbf{i}}(K)\right)+g\left(S_{\mathbf{i}}\left(x_{0}\right)\right)-g^{\prime}\left(S_{\mathbf{i}}\left(x_{0}\right)\right) \cdot\left(S_{\mathbf{i}}\left(x_{0}\right)\right), \zeta r_{\mathbf{i}} \operatorname{diam}(K)\right) .
$$

Since $\mathcal{U}_{\mathbf{i}}$ is an open cover of $g^{\prime}\left(S_{\mathbf{i}}\left(x_{0}\right)\right) \circ T_{\mathbf{i}}(K)$ it follows from (26) that

$$
\left\{B\left(r_{\mathbf{i}} U+g^{\prime}\left(S_{\mathbf{i}}\left(x_{0}\right)\right) \cdot g\left(S_{\mathbf{i}}(0)\right)+g\left(S_{\mathbf{i}}\left(x_{0}\right)\right)-g^{\prime}\left(S_{\mathbf{i}}\left(x_{0}\right)\right) \cdot\left(S_{\mathbf{i}}\left(x_{0}\right)\right), \zeta r_{\mathbf{i}} \operatorname{diam}(K)\right): U \in \mathcal{U}_{\mathbf{i}}\right\}
$$

is an open cover of $g\left(S_{\mathbf{i}}(K)\right)$. We have that

$$
\begin{gathered}
\operatorname{diam}\left(B\left(r_{\mathbf{i}} U+g^{\prime}\left(S_{\mathbf{i}}\left(x_{0}\right)\right) \cdot g\left(S_{\mathbf{i}}(0)\right)+g\left(S_{\mathbf{i}}\left(x_{0}\right)\right)-g^{\prime}\left(S_{\mathbf{i}}\left(x_{0}\right)\right) \cdot\left(S_{\mathbf{i}}\left(x_{0}\right)\right), \zeta r_{\mathbf{i}} \operatorname{diam}(K)\right)\right) \\
\leq \operatorname{diam}\left(B\left(r_{\mathbf{i}} \cdot U, \zeta r_{\mathbf{i}} \operatorname{diam}(K)\right)\right) \leq r_{\mathbf{i}}(\operatorname{diam}(U)+2 \zeta \operatorname{diam}(K)) \\
\leq r_{\mathbf{i}} \operatorname{diam}(U)(1+2 \operatorname{diam}(K)) .
\end{gathered}
$$

Since $g$ is a Lipschitz map it follows from (24) that

$$
\mathcal{H}_{\infty}^{t}\left(g\left(K \backslash\left(\bigcup_{\mathbf{i} \in \mathcal{J}} K_{\mathbf{i}}\right)\right)\right)=0 .
$$

Hence by (27)

$$
\begin{aligned}
\mathcal{H}_{\infty}^{t}(g(K)) & \leq \sum_{\mathbf{i} \in \mathcal{J}} \sum_{U \in \mathcal{U}_{\mathbf{i}}} r_{\mathbf{i}}^{t} \operatorname{diam}(U)^{t}(1+2 \operatorname{diam}(K))^{t} \\
& \leq(1+2 \operatorname{diam}(K))^{t} \sum_{\mathbf{i} \in \mathcal{J}} r_{\mathbf{i}}^{t} \sum_{U \in \mathcal{U}_{\mathbf{i}}} \operatorname{diam}(U)^{t}<(1+2 \operatorname{diam}(K))^{t} \varepsilon
\end{aligned}
$$

where we used (23) and (25). Since this is true for every $\varepsilon>0$ it follows that $\mathcal{H}_{\infty}^{t}(g(K))=0$ and hence $\mathcal{H}^{t}(g(K))=0$.

\section{Transformation groups of dense orbits and Hausdorff dimension}

In this section we prove Theorem 1.6 and Corollary [1.7. First we show Proposition 1.8 and then we derive Theorem 1.6 from [17, Corollary 1.7] and Proposition 1.8. Finally we conclude Corollary 1.7 from Theorem 1.6.

Lemma 7.1. Let $S_{1}: \mathbb{R}^{d} \longrightarrow \mathbb{R}^{d}$ and $S_{2}: \mathbb{R}^{d} \longrightarrow \mathbb{R}^{d}$ be contracting similarities with no common fixed point. Then the similarities $S_{1}^{n} \circ S_{2}$ have different fixed points for all $n \in \mathbb{N}$.

Proof. By Banach's fixed point theorem every contracting similarity $S: \mathbb{R}^{d} \longrightarrow \mathbb{R}^{d}$ has a unique fixed point. Assume for a contradiction that there exist $x \in \mathbb{R}^{d}, n \in \mathbb{N}$ and a positive integer $k$ such that that $S_{1}^{n} \circ S_{2}(x)=x$ and $S_{1}^{k} \circ S_{1}^{n} \circ S_{2}(x)=x$. Then $S_{1}^{-k}(x)=S_{1}^{n} \circ S_{2}(x)=x$. It follows that the unique fixed point of $S_{1}$ is $x$. But then $S_{2}(x)=S_{1}^{-n}(x)=x$ contradicting that $S_{1}$ and $S_{2}$ have no common fixed point. 
Lemma 7.2. Let $S_{1}, \ldots, S_{m}: \mathbb{R}^{d} \longrightarrow \mathbb{R}^{d}$ be contracting similarities $(m \geq 2)$ such that $S_{1}$ and $S_{2}$ have no common fixed point. Then there exist $F_{1}, \ldots, F_{m}: \mathbb{R}^{d} \longrightarrow \mathbb{R}^{d}$ such that $F_{1}=S_{1}, F_{2}=S_{2}$, for each $i \in\{3, \ldots, m\}$ either $F_{i}=S_{1}^{k_{i}} \circ S_{i}$ or $F_{i}=S_{2}^{k_{i}} \circ S_{i}$ for some $k_{i} \in \mathbb{N}$, and $F_{i}$ and $F_{j}$ have no common fixed point for all $i, j \in\{1, \ldots, m\}, i \neq j$.

Proof. We prove this by induction on $m$. If $m=2$ then it is trivial. Let $m>2$. Then by the inductive assumption we can find such a system $F_{1}, \ldots, F_{m-1}$ that satisfies the conclusion for $S_{1}, \ldots, S_{m-1}$. The unique fixed point of $S_{m}$ is either not the fixed point of $S_{1}$ or not the fixed point of $S_{2}$. Without the loss of generality we can assume that $S_{m}$ and $S_{1}$ have no common fixed points. Then by Lemma 7.1 there exists $k_{m} \in \mathbb{N}$ such that the fixed point of $S_{1}^{k_{m}} \circ S_{m}$ is different from the fixed points of $F_{1}, \ldots, F_{m-1}$. If we set $F_{m}=S_{1}^{k_{m}} \circ S_{m}$ then $F_{1}, \ldots, F_{m}$ satisfies the conclusion.

Now we are ready to prove Proposition 1.8. The proof consists of two steps. First we find a collection of words $\mathbf{j}_{i} \in \bigcup_{k=1}^{\infty} \mathcal{I}^{k}$ such that the group generated by $T_{\mathbf{j}_{i}}$ is dense in $\mathcal{T}$ and the $S_{\mathbf{j}_{i}}(K)$ are disjoint. At this point we do not care about the dimension. Then we add another finite set of maps to the new SS-IFS so that the strong separation condition still holds and the dimension becomes arbitrarily close to that of $K$.

Proof of Proposition 1.8. Since $K$ has at least two points there exist $i, j \in \mathcal{I}$ such that $S_{i}$ and $S_{j}$ have no common fixed point, otherwise the common fixed point would be the attractor. Without the loss of generality we can assume that $i=1$ and $j=2$.

It follows from Lemma 7.2 that there exist $\mathbf{i}_{1}, \ldots, \mathbf{i}_{m} \in \bigcup_{k=1}^{\infty} \mathcal{I}^{k}$ such that $S_{\mathbf{i}_{i}}$ and $S_{\mathbf{i}_{j}}$ have no common fixed point for all $i, j \in \mathcal{I}, i \neq j, \mathbf{i}_{1}=1, \mathbf{i}_{2}=2$ and the group generated by $T_{\mathbf{i}_{1}}, \ldots, T_{\mathbf{i}_{m}}$ is $\mathcal{T}$. Let $x_{i}$ be the unique fixed point of $S_{\mathbf{i}_{i}}$ for all $i \in \mathcal{I}$. Let $d_{\min }=$ $\min \left\{\left\|x_{i}-x_{j}\right\|: i, j \in \mathcal{I}, i \neq j\right\}>0, r_{\max }=\max \left\{r_{i}: i \in \mathcal{I}\right\}<1$ and $N \in \mathbb{N}$ such that $r_{\max }^{N} \cdot \operatorname{diam}(K)<\frac{d_{\min }}{2}$. Then $S_{\mathbf{i}_{i}}^{k_{i}}(K) \cap S_{\mathbf{i}_{j}}^{k_{j}}(K)=\emptyset$ for all $i, j \in \mathcal{I}, i \neq j, k_{i}, k_{j} \in \mathbb{N}$, $k_{i}, k_{j} \geq N$.

By Proposition 2.2 for all $i \in \mathcal{I}$ we can find $k_{i} \in N, k_{i} \geq N$ such that the group generated by $T_{\mathbf{i}_{i}}^{k_{i}}$ is dense in the group generated by $T_{\mathbf{i}_{i}}$. It follows that the group generated by $T_{\mathbf{i}_{1}}^{k_{1}}, \ldots, T_{\mathbf{i}_{m}}^{k_{m}}$ is dense in $\mathcal{T}$ and $S_{\mathbf{i}_{i}}^{k_{i}}(K) \cap S_{\mathbf{i}_{j}}^{k_{j}}(K)=\emptyset$ for all $i, j \in \mathcal{I}, i \neq j$. Let $\widehat{S}_{i}=S_{\mathbf{i}_{i}}^{k_{i}}$ for all $i \in \mathcal{I}$.

Let $F=\bigcup_{i \in \mathcal{I}} S_{\mathbf{i}_{i}}^{k_{i}}(K)$. If $K=F$ then $\left\{\widehat{S}_{i}\right\}_{i=1}^{m}$ satisfies the SSC with attractor $\widehat{K}=K$ and the proof is complete. So we can assume that $F \subsetneq K$. Let $\mathbf{j} \in \bigcup_{k=1}^{\infty} \mathcal{I}^{k}$ be such that $K_{\mathbf{j}} \cap F=\emptyset$. Let $t=\operatorname{dim}_{H} K=\operatorname{dim}_{H} K_{\mathbf{j}}$. Since $K$ has at least two points it follows that $K$ has infinitely many points but by Proposition 2.9 $\mathcal{H}^{t}(K)<\infty$, thus $t>0$ and hence without the loss of generality we can assume that $t>\varepsilon>0$. Since $\mathcal{H}^{t-\frac{\varepsilon}{2}}\left(K_{\mathbf{j}}\right)=\infty$ we can find $\delta>0$ such that for any $3 \delta$-cover $\mathcal{U}$ of $K_{\mathbf{j}}$ we have that $\sum_{U \in \mathcal{U}} \operatorname{diam}(U)^{t-\frac{\varepsilon}{2}}>1$. Let $r_{\min }=\min \left\{r_{i}: i \in \mathcal{I}\right\}<1$ and let $\mathcal{J}=\left\{\mathbf{i} \in \bigcup_{k=1}^{\infty} \mathcal{I}^{k}: K_{\mathbf{i}} \subseteq K_{\mathbf{j}}, r_{\min } \delta \leq \operatorname{diam}\left(K_{\mathbf{i}}\right)<\delta\right\}$. Then $\left\{K_{\mathbf{i}}: \mathbf{i} \in \mathcal{J}\right\}$ is a cover of $K_{\mathbf{j}}$. Let $\mathbf{j}_{1}, \ldots, \mathbf{j}_{n} \in \mathcal{J}$ be such that $K_{\mathbf{j}_{1}}, \ldots, K_{\mathbf{j}_{n}}$ is a maximal pairwise disjoint sub-collection of $\left\{K_{\mathbf{i}}: \mathbf{i} \in \mathcal{J}\right\}$. Let $U_{j}$ be the $\delta$-neighbourhood of $K_{\mathbf{j}_{j}}$ for $j \in\{1, \ldots, n\}$. By the maximality $\left\{U_{j}: j \in\{1, \ldots, n\}\right\}$ is a $3 \delta$-cover of $K_{\mathbf{j}}$. Hence by the choice of $\delta$

$$
\sum_{j=1}^{n}(3 \delta)^{t-\frac{\varepsilon}{2}} \geq \sum_{j=1}^{n}\left(\operatorname{diam}\left(U_{j}\right)\right)^{t-\frac{\varepsilon}{2}}>1 .
$$


It follows that $n \geq(3 \delta)^{-\left(t-\frac{\varepsilon}{2}\right)}$. Let $K_{0}$ be the attractor of the SS-IFS $\left\{S_{\mathbf{j}_{j}}\right\}_{j=1}^{n}$. Then $K_{0} \subseteq K$, the SS-IFS $\left\{S_{\mathbf{j}_{j}}\right\}_{j=1}^{n}$ satisfies the SSC and

$$
\operatorname{dim}_{H} K_{0} \geq \frac{\log \left(\frac{1}{n}\right)}{\log \left(\frac{r_{\min } \cdot \delta}{\operatorname{diam}(K)}\right)} \geq \frac{-\left(t-\frac{\varepsilon}{2}\right) \cdot \log (3)-\left(t-\frac{\varepsilon}{2}\right) \cdot \log (\delta)}{\log (\operatorname{diam}(K))-\log \left(r_{\min }\right)-\log (\delta)}
$$

because the similarity dimension of $\left\{S_{\mathbf{j}_{j}}\right\}_{j=1}^{n}$ is $\operatorname{dim}_{H} K_{0}$ by Proposition 2.10, So, by choosing $\delta$ small enough, $\operatorname{dim}_{H} K_{0}>t-\varepsilon$. Let $\widehat{m}=m+n, \widehat{S_{m+j}}=S_{\mathbf{j}_{j}}$ for all $j \in\{1, \ldots, n\}$ and $\widehat{K}$ be the attractor of the SS-IFS $\left\{\widehat{S}_{i}\right\}_{i=1}^{\widehat{m}}$. Then the transformation group $\widehat{\mathcal{T}}$ of $\left\{\widehat{S}_{i}\right\}_{i=1}^{\widehat{m}}$ is dense in $\mathcal{T}, K_{0} \subseteq \widehat{K} \subseteq K, \operatorname{dim}_{H} K-\varepsilon<\operatorname{dim}_{H} K_{0} \leq \operatorname{dim}_{H} \widehat{K}$ and $\left\{\widehat{S}_{i}\right\}_{i=1}^{\widehat{m}}$ satisfies the SSC.

A similar argument to the last step of the proof of Proposition 1.8 was used in the proof of [28, Theorem 2].

Proof of Theorem 1.6. The upper bound $\operatorname{dim}_{H}(g(K)) \leq \min \{t, l\}$ follows since $g$ is a Lipschitz map on $K$.

First assume that $\operatorname{rank}\left(g^{\prime}(x)\right)=l$ holds for every $x \in U$. By Proposition 1.8, for all $\varepsilon>0$ there exists an SS-IFS $\left\{\widehat{S}_{i}\right\}_{i=1}^{\widehat{m}}$ that satisfies the SSC with attractor $\widehat{K}$ such that $\widehat{K} \subseteq K, \operatorname{dim}_{H} K-\varepsilon<\operatorname{dim}_{H} \widehat{K}$ and for the transformation group $\widehat{\mathcal{T}}$ of $\left\{\widehat{S}_{i}\right\}_{i=1}^{\widehat{m}}$ we have that $\{O(M): O \in \widehat{\mathcal{T}}\}$ is dense in $G_{d, l}$. By [17, Corollary 1.7] $\operatorname{dim}_{H}(g(\widehat{K}))=\min \left\{\operatorname{dim}_{H} \widehat{K}, l\right\}$. Hence

$$
\operatorname{dim}_{H}(g(K)) \geq \operatorname{dim}_{H}(g(\widehat{K}))=\min \left\{\operatorname{dim}_{H} \widehat{K}, l\right\} \geq \min \left\{\operatorname{dim}_{H} K-\varepsilon, l\right\} .
$$

So $\operatorname{dim}_{H}(g(K)) \geq \min \{t-\varepsilon, l\}$ for all $\varepsilon>0$ and hence $\operatorname{dim}_{H}(g(K))=\min \{t, l\}$.

In the general case there exists $x \in K$ such that $\operatorname{rank}\left(g^{\prime}(x)\right)=l$ it follows that there exists an open neighbourhood $V$ of $x$ such that $\operatorname{rank}\left(g^{\prime}(y)\right)=l$ for every $y \in V$. For large enough $k$ there exists $\mathbf{i} \in \mathcal{I}^{k}$ such that $K_{\mathbf{i}} \subseteq V$. Then $K_{\mathbf{i}}$ is the attractor of the SS-IFS $\left\{S_{\mathbf{i}} \circ S_{j} \circ S_{\mathbf{i}}^{-1}\right\}_{j=1}^{m}$ and $\left\{O(M): O \in \mathcal{T}_{\mathbf{i}}\right\}$ is dense in $G_{d, l}$ where $\mathcal{T}_{\mathbf{i}}$ is the transformation group of $\left\{S_{\mathbf{i}} \circ S_{j} \circ S_{\mathbf{i}}^{-1}\right\}_{j=1}^{m}$. Thus we can assume that $\operatorname{rank}\left(g^{\prime}(x)\right)=l$ holds for every $x \in U$.

Proof of Corollary 1.7. Let $g(y)=\left(g_{1}(y), \ldots, g_{d_{2}}(y)\right)$ and set an arbitrary point $x \in K$. Since $\operatorname{rank}\left(g^{\prime}(x)\right)=l$ it follows that there are $l$ coordinate indices $1 \leq j_{1} \leq \ldots \leq j_{l} \leq d_{2}$ such that the vectors $g_{j_{1}}^{\prime}(x), \ldots, g_{j_{l}}^{\prime}(x)$ are linearly independent. Let $P: \mathbb{R}^{d_{2}} \longrightarrow \mathbb{R}^{l}$ be the projection $P(y)=\left(y_{j_{1}}, \ldots, y_{j_{l}}\right)$ and $f: U \longrightarrow \mathbb{R}^{l}$ be $f(y)=P \circ g(y)$. Note that $P$ and hence $f$ may depend on $x$. Then the conditions of Theorem 1.6 are satisfied for $f$ in place of $g$. Thus $\operatorname{dim}_{H} P \circ g(K)=\min \{t, l\}$ and hence $\operatorname{dim}_{H} g(K) \geq \min \{t, l\}$.

The upper bound in case of $i$ ) follows from [11, Theorem 3.4.3]. The upper bound in case of $i$ ) follows since $g$ is a Lipschitz map on $K$ and hence $\operatorname{dim}_{H} g(K) \leq t=\min \{t, l\}$. 


\section{Examples and questions}

In this section we raise some open questions and provide examples.

If the Hausdorff dimension coincides with the similarity dimension and $|\mathcal{T}|<\infty$ then by Theorem 1.2 there must be at least one projection where the dimension drops. The following example is very well-known and shows that it is possible to have a projection of positive measure when $|\mathcal{T}|<\infty$.

Example 8.1. For $0<t \leq 1$ the $t$-dimensional Sierpinski triangle is the attractor of the SS-IFS that contains three homotheties which map an equilateral triangle into itself fixing the corners with similarity ratio $r=3^{-1 / t}$. Then $|\mathcal{T}|=1$ and $\mathcal{H}^{t}\left(\Pi_{M}(K)\right)>0$ where $M$ is a line paralell to one of the sides of the triangle.

Question 8.2. Is it true that if $|\mathcal{T}|<\infty$ and $t \leq l<d$ then we can always find $l$ dimensional subspaces $M_{1}$ and $M_{2}$ such that $\mathcal{H}^{t}\left(\Pi_{M_{1}}(K)\right)>0$ and $\operatorname{dim}_{H}\left(\Pi_{M_{2}}(K)\right)<t$ ?

Theorem 1.5 shows that if $\{O(M): O \in \mathcal{T}\}$ is dense in $G_{d, l}$ for some $M \in G_{d, l}$ then every projection is of zero measure, on the other hand Theorem 1.6 shows that there is no projection where the dimension drops. Example 8.3 shows that $|\mathcal{T}|=\infty$ is not enough to imply either of these results.

Example 8.3. There exists a self-similar set $K$ with $t=\operatorname{dim}_{H}(K)$ such that $|\mathcal{T}|=\infty$ and there exist three different orthogonal projections $P_{1}, P_{2}, P_{3}$ of $K$ onto lines with the following properties: $t=\operatorname{dim}_{H}\left(P_{1}\right)$ and $\mathcal{H}^{t}\left(P_{1}\right)=0, \mathcal{H}^{t}\left(P_{2}\right)>0$ and $\operatorname{dim}_{H}\left(P_{3}\right)<t$. Let $T_{1}: \mathbb{R}^{2} \longrightarrow \mathbb{R}^{2}$ be a rotation around the origin by angle $\alpha \cdot \pi$ for some $\alpha \notin \mathbb{Q}$ and let $T$ : $\mathbb{R}^{2} \times \mathbb{R}^{2} \longrightarrow \mathbb{R}^{2} \times \mathbb{R}^{2}$ be defined as $T(x, y)=\left(T_{1}(x), y\right)$ for $x, y \in \mathbb{R}^{2}$. Let $r \leq \frac{1}{3}$ and $v_{1, i} \in \mathbb{R}^{2}$ for $i=1,2,3$ be such that the SS-IFS $\left\{r \cdot T_{1}(x)+v_{1, i}\right\}_{i=1}^{3}$ satisfies the SSC with attractor $K_{1}$. Let $v_{2, i} \in \mathbb{R}^{2}$ for $i=1,2,3$ be such that the attractor of the SS-IFS $\left\{r \cdot I d_{\mathbb{R}^{2}}(x)+v_{2, i}\right\}_{i=1}^{3}$ is the $\frac{\log (3)}{\log \left(r^{-1}\right)}$-dimensional Sierpinski triangle $K_{2}$. Set $v_{i}=\left(v_{1, i}, v_{2, i}\right) \in \mathbb{R}^{2} \times \mathbb{R}^{2}, S_{i}(z)=$ $r \cdot T(z)+v_{i}$ for $z \in \mathbb{R}^{2} \times \mathbb{R}^{2}, i=1,2,3$ and let $K$ be the attractor of the SS-IFS $\left\{S_{i}\right\}_{i=1}^{3}$. Then $\left\{S_{i}\right\}_{i=1}^{3}$ satisfies the SSC, hence $t=\operatorname{dim}_{H} K=\operatorname{dim}_{H} K_{1}=\operatorname{dim}_{H} K_{2}=\frac{\log (3)}{\log \left(r^{-1}\right)}$. Let $M_{1}=\mathbb{R}^{2} \times(0,0)$, let $L_{1} \subseteq M_{1}$ be any line, $M_{2}=(0,0) \times \mathbb{R}^{2}$ and $L_{2}=(0,0) \times \mathbb{R} \times(0)$. One can show that $\Pi_{M_{1}}(K)=K_{1}$, thus $P_{1}=\Pi_{L_{1}}(K)=\Pi_{L_{1}} \circ \Pi_{M_{1}}(K)=\Pi_{L_{1}}\left(K_{1}\right)$ and hence by Theorem 1.6 and Theorem 1.5 $\operatorname{dim}_{H}\left(\Pi_{L_{1}}(K)\right)=t$ and $\mathcal{H}^{t}\left(\Pi_{L_{1}}(K)\right)=0$. On the other hand $\Pi_{M_{2}}(K)=K_{2}$, thus $P_{2}=\Pi_{L_{2}}(K)=\Pi_{L_{2}} \circ \Pi_{M_{2}}(K)=\Pi_{L_{2}}\left(K_{2}\right)$ and hence $\mathcal{H}^{t}\left(\Pi_{L_{2}}(K)\right)>0$. Finally by Theorem 1.2 there exists a line $L_{3} \subseteq M_{2}$ such that $\operatorname{dim}_{H}\left(\Pi_{L_{3}}\left(K_{2}\right)\right)<t$ and hence $\operatorname{dim}_{H}\left(\Pi_{L_{3}}(K)\right)<t$. The transformation group $\mathcal{T}$ of $\left\{S_{i}\right\}_{i=1}^{3}$ is infinite, but $\{O(L): O \in \mathcal{T}\}$ is not dense in $G_{4,1}$ for any $L \in G_{4,1}$ and $K$ is not contained in any affine hyperplane.

Is there a counterexample to the converse of Proposition 6.2 and Theorem 1.6?

Question 8.4. Is there an example of an $S S$-IFS such that $\mathcal{T}$ is infinite, $\operatorname{dim}_{H}\left(\Pi_{N}(K)\right)=t$ and $\mathcal{H}^{t}\left(\Pi_{N}(K)\right)=0$ for every $N \in G_{d, l}$, but $\{O(M): O \in \mathcal{T}\}$ is not dense in $G_{d, l}$ for any $M \in G_{d, l}$ and $\mathcal{H}^{t}(K)>0$ ?

The following example shows that we cannot replace $g$ with a Lipschitz function in Theorem 1.6 and Theorem 1.5 . 
Example 8.5. Let $t \leq 1$ and $\left\{S_{i}\right\}_{i=1}^{3}$ be an SS-IFS in $\mathbb{R}^{2}$ such that $S_{1}$ and $S_{2}$ are two maps from the usual SS-IFS of the $t$-dimensional Sierpinski triangle and we slightly modify the orthogonal part of the third map so that $T_{3}$ is a rotation of a small angle $\alpha \cdot \pi$ for some $\alpha \notin \mathbb{Q}$. Let $K$ be the attractor of $\left\{S_{i}\right\}_{i=1}^{3}$ and $\widehat{K}$ be the $t$-dimensional Sierpinski triangle. Then one can show that the natural bijection $f$ between $K$ and $\widehat{K}$ is a bi-Lipschitz function. Then the assumptions of Theorem 1.5 holds for $\left\{S_{i}\right\}_{i=1}^{3}$ and $l=1$ but there exist lines $M_{1}$ and $M_{2}$ such that $\mathcal{H}^{t}\left(\Pi_{M_{1}}(f(K))\right)>0$ and $\operatorname{dim}_{H}\left(\Pi_{M_{2}}(f(K))\right)<t$.

The two following examples show that the assumption $\mathcal{H}^{t}(K)>0$ where $t=\operatorname{dim}_{H} K$ is weaker than the OSC.

Example 8.6. There exists a self-similar set $\widehat{K} \subseteq \mathbb{R}$ such that no SS-IFS with attractor $\widehat{K}$ satisfies the OSC but $0<\mathcal{H}^{t}(\widehat{K})<\infty$ where $t=\operatorname{dim}_{H} \widehat{K}$.

Let $0<r<\frac{1}{3}$ and $g=\frac{1-3 r}{2}$. We first define an SS-IFS as follows (see Figure 1): $S_{1}(x)=r \cdot x, S_{2}(x)=r \cdot x+r+g$ and $S_{3}(x)=r \cdot x+2 r+2 g$. We denote by $K$ the attractor of $\left\{S_{i}\right\}_{i=1}^{3}$. Since $\left\{S_{i}\right\}_{i=1}^{3}$ satisfies the OSC it follows that $0<\mathcal{H}^{t}(K)<\infty$ where $t=\operatorname{dim}_{H} K$. The set $\widehat{K}=K \backslash S_{3}(K)=S_{1}(K) \cup S_{2}(K)$ is also a self similar set, namely it is the attractor of an SS-IFS containing the following four maps: $\widehat{S}_{1}(x)=S_{1}(x)$, $\widehat{S}_{2}(x)=S_{1}(x)+r(r+g), \widehat{S}_{3}(x)=S_{2}(x)$ and $\widehat{S}_{4}(x)=S_{2}(x)+r(r+g)$. We have that $0<\mathcal{H}^{t}(\widehat{K})<\infty$.

Let $F(x)=a \cdot x+b$ a contractive similarity such that $F(\widehat{K}) \subseteq \widehat{K}$. We show that $a=r^{n}$ for some positive integer $n$. We call the length of the longest bounded component of the complement of a compact set the largest gap.

First assume that $r \leq a<1$. The largest gap of $\widehat{K}$ is $g$ and the largest gap of $F(\widehat{K})$ is $a g<g$. The distance between $S_{1}(K)$ and $S_{2}(K)$ is $g$ hence either $F(\widehat{K}) \subseteq S_{1}(K)$ or $F(\widehat{K}) \subseteq S_{2}(K)$. For simplicity assume that $F(\widehat{K}) \subseteq S_{1}(K)$, the proof goes similarly in the case $F(\widehat{K}) \subseteq S_{2}(K)$. The largest gap of $F \circ S_{1}(K)$ is arg $<r g$. The smallest distance between the sets $S_{1} \circ S_{1}(K), S_{1} \circ S_{2}(K)$ and $S_{1} \circ S_{3}(K)$ is $r g$. Hence either $F \circ S_{1}(K) \subseteq S_{1} \circ S_{1}(K)$ or $F \circ S_{1}(K) \subseteq S_{1} \circ S_{2}(K)$ or $F \circ S_{1}(K) \subseteq S_{1} \circ S_{3}(K)$. Thus ar $\leq r r$ and so $a \leq r$. Since we assumed $r \leq a<1$ it follows that $a=r$.

Now assume that $r^{n} \leq a<r^{n-1}$ for some positive integer $n$. As above we can show that $F(\widehat{K}) \subseteq S_{\mathbf{i}}(K)$ for some $\mathbf{i} \in\{1,2,3\}^{n}$ and $F \circ S_{1}(K) \subseteq S_{\mathbf{i}} \circ S_{j}(K)$ for some $j \in\{1,2,3\}$. Hence $a=r^{n}$.

Since $F(\widehat{K}) \subseteq S_{\mathbf{i}}(K)$ for some $\mathbf{i} \in\{1,2,3\}^{n}$ and $a=r^{n}$ it follows that either

$$
F(\widehat{K})=S_{\mathbf{i}} \circ S_{1}(K) \cup S_{\mathbf{i}} \circ S_{2}(K) \text { or } F(\widehat{K})=S_{\mathbf{i}} \circ S_{2}(K) \cup S_{\mathbf{i}} \circ S_{3}(K)
$$

because the largest gap of $F(\widehat{K})$ and one of the largest gaps of $S_{\mathbf{i}}(K)$ must coincide.

Let $\left\{F_{i}\right\}_{i=1}^{m}$ be an SS-IFS with attractor $\widehat{K}$. Without the loss of generality we can assume that the similarity ratio $r^{n}$ of $F_{1}(x)$ is the smallest of the similarity ratios of the maps $F_{i}$. By (28) and the minimality of $r^{n}$ there exists $j \in\{1, \ldots, m\}$ such that $S_{\mathbf{i}}(K) \backslash$ $F_{1}(\widehat{K}) \subseteq F_{j}(\widehat{K})$ and either $F_{1}(\widehat{K}) \cap F_{j}(\widehat{K})=F_{1}(\widehat{K})$ or $F_{1}(\widehat{K}) \cap F_{j}(\widehat{K})=S_{\mathbf{i}} \circ S_{2}(K)$. Thus $\mathcal{H}^{t}\left(F_{1}(\widehat{K}) \cap F_{j}(\widehat{K})\right)>0$ and so $\left\{F_{i}\right\}_{i=1}^{m}$ cannot satisfy the OSC. 


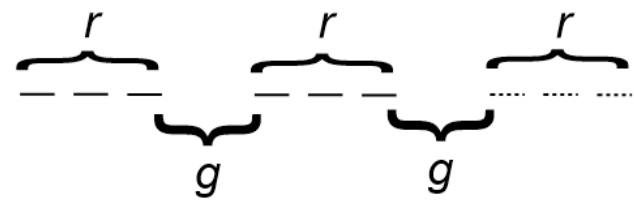

Figure 1.

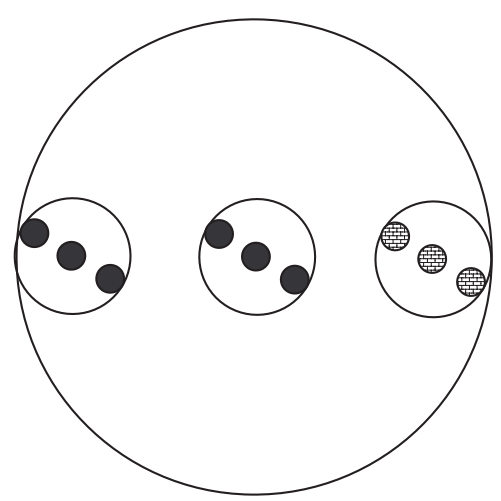

Figure 2.

Example 8.7. Let $T$ be a rotation around the origin by angle $\alpha \in[0,2 \pi)$. There exists a self-similar set $\widehat{K} \subseteq \mathbb{R}^{2}$ such that no SS-IFS with attractor $\widehat{K}$ satisfies the OSC but $0<\mathcal{H}^{t}(\widehat{K})<\infty$ where $t=\operatorname{dim}_{H} \widehat{K}$ and there exists an SS-IFS with attractor $\widehat{K}$ such that the transformation group of the SS-IFS is generated by $T$.

Let $0<r<\frac{1}{3}$ and $g=1-3 r$. We define an SS-IFS in $\mathbb{R}^{2}$ as follows (see Figure 2): $S_{1}(x)=r T(x)+(-g-2 r, 0), S_{2}(x)=r T(x)$ and $S_{3}(x)=r T(x)+(g+2 r, 0)$. We denote by $K$ the attractor of $\left\{S_{i}\right\}_{i=1}^{3}$. Since $\left\{S_{i}\right\}_{i=1}^{3}$ satisfies the OSC it follows that $0<\mathcal{H}^{t}(K)<\infty$ where $t=\operatorname{dim}_{H} K$. The set $\widehat{K}=K \backslash S_{3}(K)=S_{1}(K) \cup S_{2}(K)$ is also a self-similar set, namely it is the attractor of an SS-IFS containing the following four maps: $\widehat{S}_{1}(x)=S_{1}(x)$, $\widehat{S_{2}}(x)=S_{1}(x+(g+2 r, 0)), \widehat{S}_{3}(x)=S_{2}(x)$ and $\widehat{S}_{4}(x)=S_{2}(x+(g+2 r, 0))$. We have that $0<\mathcal{H}^{t}(\widehat{K})<\infty$ and the transformation group of $\left\{\widehat{S}_{i}\right\}_{i=1}^{4}$ is generated by $T$.

We can show that there is no SS-IFS with attractor $\widehat{K}$ that satisfies the OSC via a similar argument to the argument in Example 8.6 with the difference that the largest gap of $K$ and $\widehat{K}$ will be replaced by the smallest distance between $S_{1}(K)$ and $S_{2}(K)$. We note that this distance is greater than $g$.

Remark 8.8. We note that both in Example 8.6 and Example 8.7 the semigroup generated by $\left\{\widehat{S}_{i}\right\}_{i=1}^{4}$ is not free. Hence after iteration and deleting repetitions one can reduce the similarity dimension of the SS-IFS. It is not hard to see that we can find an SS-IFS with attractor $\widehat{K}$ of similarity dimension arbitrarily close to $t$ but we cannot find an SS-IFS with attractor $\widehat{K}$ of similarity dimension $t$ because of Proposition 2.10.

\section{Acknowledgements}

The author was supported by an EPSRC doctoral training grant and thanks Kenneth Falconer, Jonathan Fraser and the anonymous referee for many valuable suggestions on improving the exposition of the article.

\section{References}

[1] R. B. Bapat and T. E. S. Raghavan, Nonnegative Matrices and Applications, Cambridge University Press, Cambridge, 1997. MR1449393 (98h:15038) 
[2] A. S. Besicovitch, On the fundamental geometrical properties of linearly measurable plane sets of points (III), Math. Ann. 116 (1939), 349-357. MR1513231

[3] T. S. Blyth and E. F. Robertson, Further Linear Algebra, Springer-Verlag London, Ltd., London, 2002. MR1864441

[4] J. C. Burkill and H. Burkill, A Second Course in Mathematical Analysis, Cambridge University Press, London-New York, 1970. MR0258550 (41 \#3197)

[5] G. A. Edgar, Measure, Topology, and Fractal Geometry, Springer-Verlag, New York, 1990. MR1065392 (92a:54001)

[6] K. I. Eroğlu, On planar self-similar sets with a dense set of rotations, Ann. Acad. Sci. Fenn. Math. 32 (2007), 409-424. MR2337485 (2009c:28014)

[7] K. Falconer, Fractal Geometry, Third edition. John Wiley \& Sons, Ltd., Chichester, 2014.

[8] K. Falconer, Techniques in Fractal Geometry, John Wiley \& Sons, Ltd., Chichester, 1997. MR1449135 (99f:28013)

[9] K. J. Falconer, The Geometry of Fractal Sets, Cambridge University Press, Cambridge, 1986. MR0867284 (88d:28001)

[10] K. Falconer and X. Jin, Exact dimensionality and projections of random self-similar measures and sets, J. London Math. Soc. (2) 90 (2014), 388-412.

[11] H. Federer, Geometric Measure Theory, Springer-Verlag New York Inc., New York, 1969. MR0257325 (41 \#1976)

[12] H. Federer, The $(\varphi, k)$ rectifiable subsets of $n$-space, Trans. Amer. Soc. 62, (1947), 114-192. MR0022594 (9,231c)

[13] J. M. Fraser, On the packing dimension of box-like self-affine sets in the plane, Nonlinearity 25 (2012), 2075-2092. MR2947936

[14] H. Furstenberg, Ergodic fractal measures and dimension conservation, Ergodic Theory Dynam. Systems 28 (2008), 405-422. MR2408385 (2009e:28032)

[15] G. H. Hardy and E. M. Wright, An Introduction to the Theory of Numbers, Oxford, at the Clarendon Press, 1954. MR0067125 (16,673c)

[16] M. Hochman, On self-similar sets with overlaps and inverse theorems for entropy, Ann. of Math. (2) 180 (2014), 773-822. MR3224722

[17] M. Hochman and P. Shmerkin, Local entropy averages and projections of fractal measures, Ann. of Math. (2) 175 (2012), 1001-1059. MR2912701

[18] J. E. Hutchinson, Fractals and self-similarity, Indiana Univ. Math. J. 30 (1981), 713-747. MR0625600 (82h:49026) 
[19] K. Simon and B. Solomyak, Visibility for self-similar sets of dimension one in the plane, Real Anal. Exchange 32 (2006/07), 67-78. MR2329222

[20] R. Kenyon, Projecting the one-dimensional Sierpinski gasket. Israel J. Math. 97 (1997), 221-238. MR1441250 (98i:28002)

[21] M. Marcus and H. Minc, A Survey of Matrix Theory and Matrix Inequalities, Dover Publications, Inc., New York, 1992. MR1215484

[22] J. M. Marstrand, Some fundamental geometrical properties of plane sets of fractional dimensions, Proc. London Math. Soc. (3) 4 (1954), 257-302. MR0063439 (16,121g)

[23] P. Mattila, Geometry of Sets and Measures in Euclidean Spaces, Cambridge University Press, Cambridge, 1995. MR1333890 (96h:28006)

[24] P. Mattila, Hausdorff dimension, orthogonal projections and intersections with planes, Ann. Acad. Sci. Fenn. Ser. A I Math. 1 (1975), 227-244. MR0409774 (53 \#13526)

[25] P. Mattila, Hausdorff dimension, projections, and the Fourier transform, Publ. Mat. 48 (2004), 3-48. MR2044636 (2004k:28018)

[26] R. D. Mauldin and S. C. Williams, Hausdorff dimension in graph directed constructions, Trans. Amer. Math. Soc. 309 (1988), 811-829. MR0961615 (89i:28003)

[27] T. Orponen, On the distance sets of self-similar sets, Nonlinearity 25 (2012), 1919-1929. MR2929609

[28] Y. Peres and P. Shmerkin, Resonance between Cantor sets, Ergodic Theory Dynam. Systems 29 (2009), 201-221. MR2470633 (2010a:28015)

[29] Y. Peres, K. Simon and B. Solomyak, Self-similar sets of zero Hausdorff measure and positive packing measure, Israel J. Math. 117 (2000), 353-379. MR1760599 (2001g:28017)

[30] A. Schief, Separation properties for self-similar sets, Proc. Amer. Math. Soc. 122 (1994), 111-115. MR1191872 (94k:28012)

[31] JingLing Wang, The open set conditions for graph directed self-similar sets, Random Comput. Dynam. 5 (1997), 283-305. MR1483871 (99g:28019) 